\title{
REALIZING HOMOTOPY GROUP ACTIONS
}

\author{
DAVID BLANC AND DEBASIS SEN
}

\begin{abstract}
For any finite group $G$, we define the notion of a Bredon homotopy action of $G$, modelled on the diagram of fixed point sets $\left(\mathbf{X}^{H}\right)_{H \leq G}$ for a $G$ space $\mathbf{X}$, together with a pointed homotopy action of the group $N_{G} H / H$ on $\mathbf{X}^{H} /\left(\bigcup_{H<K} \mathbf{X}^{K}\right)$. We then describe a procedure for constructing a suitable diagram $\underline{X}: \mathcal{O}_{G}^{\text {op }} \rightarrow \mathcal{T}$ op from this data, by solving a sequence of elementary lifting problems. If successful, we obtain a $G$-space $\mathbf{X}^{\prime}$ realizing the given homotopy information, determined up to Bredon $G$-homotopy type. Such lifting methods may also be used to understand other homotopy questions about group actions, such as transferring a $G$-action along a map $f: \mathbf{X} \rightarrow \mathbf{Y}$.
\end{abstract}

\section{INTRODUCTION}

The naive notion of a homotopy action of a group $G$ on a topological space $\mathbf{X}$ can be described as the choice of a homotopy class of a map $\mathbf{B} G \rightarrow \mathbf{B}$ haut $(\mathbf{X})$, where $\operatorname{haut}(\mathbf{X})$ is the monoid of self-homotopy equivalences (see 91.1 ). This always lifts to a strict action, unique up to Borel equivalence (see \$1.8). However, the $G$-actions we obtain in this way will be free, so the more delicate aspects of equivariant topology are not visible in this way.

A more informative approach to equivariant homotopy theory, due to Bredon, studies $G$-spaces $\mathrm{X}$ up to $G$-homotopy equivalence - that is, $G$-maps having $G$ homotopy inverses (see $[\mathrm{Br}]$ ). This is equivalent to the homotopy theory of diagrams $\underline{\mathrm{X}}: \mathcal{O}_{G}^{\text {op }} \rightarrow \mathcal{T}$ op $\quad$ (where $\mathcal{O}_{G}$ is the orbit category of $G$ and $\underline{\mathrm{X}}(G / H)$ is the fixed point set $\mathbf{X}^{H}-$ see $\$ 1.4$ and $[\mathrm{E}]$ ). Dwyer and Kan showed that this in turn is equivalent to a homotopy theory of a certain diagram of fibrations (see [DK1, DK2]).

The purpose of this paper is to define a notion of homotopy action in Bredon equivariant homotopy theory, and describe an associated inductive procedure for realizing such an action by a continuous one.

One might be tempted to say that a homotopy action of $G$ should simply be a homotopy-commutative diagram $\underline{\mathrm{X}}^{\prime}: \mathcal{O}_{G}^{\text {op }} \rightarrow$ ho $\mathcal{T}_{o p}$. We then have available the obstruction theory of Dwyer, Kan, and Smith for rectifying general homotopycommutative diagrams (cf. [DKS2, DK3]), which we can use to try to lift $\underline{X}^{\prime}$ to a strict diagram $\underline{\mathrm{X}}: \mathcal{O}_{G}^{\mathrm{op}} \rightarrow \mathcal{T} o p$, yielding a $G$-space, unique up to Bredon equivalence.

However, the orbit category $\mathcal{O}_{G}$ can be quite complicated: it includes various isomorphisms $G / H \cong G / H^{a}$ for $a \in G$, and in particular an action of $N_{G} H$ as the automorphisms of $G / H$ for each $H \leq G$. In the Dwyer-Kan-Smith approach, all the morphisms of $\mathcal{O}_{G}$ are treated on an equal footing, and must all be made to

Date: October 17, 2018.

2010 Mathematics Subject Classification. Primary: 55P91; secondary: 55S35, 55R35, 58E40.

Key words and phrases. Group actions, equivariant homotopy type, Bredon theory, obstructions, homotopy actions. 
fit together at one time (with increasing levels of coherence). In particular, it does not allow us to interpret the initial data in terms of homotopy actions of each $N_{G} H$ on $\underline{\mathrm{X}}^{\prime}(G / H)$.

The version of homotopy action that we define here involves an ordinary diagram of spaces (with no group actions), which we assume for simplicity to be strict. We do require a certain amount of equivariant rectification in addition, but we keep this to the minimum, and in a form that reduces to an elementary lifting problem, in the spirit of [C] and [DK2], starting with certain ordinary homotopy actions of $N_{G} H$.

0.1. Bredon homotopy actions. We let $\Lambda$ denote the partially ordered set of subgroups of $G$, and define a Bredon homotopy action of $G$ to consist of:

- A diagram $\underset{\sim}{X}: \Lambda^{\mathrm{op}} \rightarrow \mathcal{T} o p$;

- For each conjugacy class $\langle H\rangle$, a pointed homotopy action of $W_{H}:=N_{G} H / H$ on the homotopy cofiber $\underset{\sim}{\mathbf{X}_{H}^{H}}$ of the obvious map hocolim $K>H \underset{\sim}{X}(K) \rightarrow$ $\underset{\sim}{X}(H)$ for some representative $H \in\langle H\rangle$.

If $H^{\prime}$ and $H$ are conjugate in $G$, we must have a homotopy-commuting square:

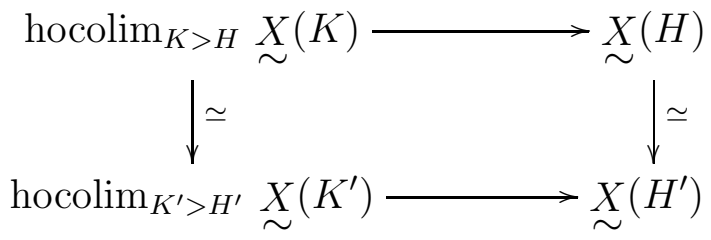

with vertical homotopy equivalences.

0.3. Realizing Bredon homotopy actions. We wish to realize such a Bredon homotopy action by a topological action, using descending induction on the subgroups of $G$ : without specifying the $G$-space $\mathbf{X}$ itself, assume that for some $H \leq G$ we have constructed a partial diagram $\underline{\mathrm{X}}$ consisting of spaces $\underline{\mathrm{X}}(K) \simeq \underset{\sim}{X}(K) \quad$ (to be thought of as of "fixed point sets" $\mathbf{X}^{K}$ for the putative $G$-space $\mathbf{X}$ ) for all groups $H<K \leq G$, together with inclusions $i^{*}: \underline{\mathrm{X}}(L) \hookrightarrow \underline{\mathrm{X}}(K)$ for $i: K \hookrightarrow L$, compatible with an action of $G$ on $\underline{\mathrm{X}}$ by $\underline{\mathrm{X}}(K) \mapsto \underline{\mathrm{X}}\left(K^{a}\right)$.

Note that we may filter the collection of subgroups of $G$ (or the objects of $\mathcal{O}_{G}$ ) by letting $\mathcal{F}_{k} \quad$ consist of those subgroups $H$ for which there is a chain of proper inclusions $H=H_{0}<H_{1}<\ldots<H_{k}=G$. If we set $\underline{\mathbf{X}}_{H}:=\bigcup_{H<K} \underline{\mathrm{X}}(K)$, by induction on this filtration we assume that we have actions of $W_{H}$ on $\underline{\mathbf{X}}_{H}$ and $\underline{\mathbf{X}}_{H}^{H}$ (the latter realizing the given pointed homotopy action on $\underset{\mathbf{X}_{H}^{H}}{\mathbf{X}_{H}}$ - see Appendix). These fit into a homotopy cofibration sequence:

$$
\underline{\mathbf{X}}_{H} \rightarrow \underset{\sim}{X}(H) \rightarrow \underline{\mathbf{X}}_{H}^{H}
$$

The key ingredient in the inductive procedure for realizing a Bredon homotopy action

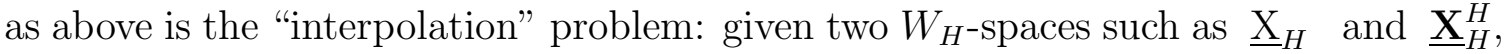
and two maps as in (0.4), how to obtain a compatible $W_{H}$-action on the middle space $\underset{\sim}{X}(H)$. This can be reduced to a lifting problem (see Propositions [3.6 and 3.10). If we succeed in solving it, we have extended our diagram $\underline{X}$ to $H$, too. 
Our main result shows that if this procedure can be completed for all $H \leq G$, we obtain a full $\mathcal{O}_{G}^{\text {op }}$-diagram $\underline{\mathrm{X}}$, and thus a $G$-space $\mathbf{X}$ realizing the given Bredon homotopy action (cf. [E]):

Theorem A. A Bredon homotopy action $\mathcal{A}:=\left\langle\underset{\sim}{X},\left(\Phi_{H}^{*}\right)_{H \leq G}\right\rangle$ for a finite group $G$ can be realized by a $G$-space $\mathbf{X}$ if and only if one can inductively construct a sequence of cofibrant diagrams $\left(\underline{X}_{k}: \mathcal{F}_{k} \rightarrow \mathcal{T}\right.$ op $)$ realizing $\mathcal{A}$. Moreover, one can extend $\underline{X}_{k}$ to $\underline{X}_{k+1}$ if and only if for each $H \subseteq \mathcal{F}_{k+1} \backslash \mathcal{F}_{k}$, one can find a map $\Psi_{H}$ making the following diagram of topological spaces commute:

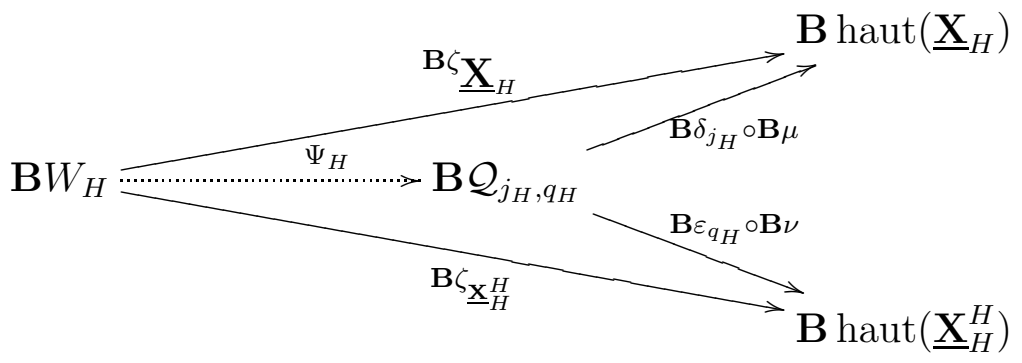

[See Theorem 4.13 below; the topological monoid $\mathcal{Q}_{f, g}$ is defined in \$3.4].

0.5. Related lifting problems. Along the way we discuss three related but simpler questions, of independent interest, and show how they too may be reduced to lifting problems for appropriate fibrations:

(i) How to extend a $G$-action on a space $\mathbf{X}$ along a map $f: \mathbf{X} \rightarrow \mathbf{Y}$;

(ii) How to lift a $G$-action on $\mathbf{Y}$ along a map $f: \mathbf{X} \rightarrow \mathbf{Y}$;

(iii) How to make a map $f: \mathbf{X} \rightarrow \mathbf{Y}$ between two $G$-spaces into a $G$-map.

See Propositions 2.7 and 2.17

0.6. Obstructions. All these lifting problems have associated obstruction theories, described in terms of (Moore-)Postnikov towers (see Proposition 3.14), and thus similar in spirit to Cooke's original approach to the realization problem for homotopy actions (see [C]). These differ from the obstruction theory of [DKS2], though unfortunately neither version is easily computable.

We may thus conclude from Theorem A that the obstructions of Proposition 3.14 are the only ones to realizing a Bredon homotopy action, and the difference obstructions distinguish between the resulting realizations up to $G$-homotopy equivalence (see Corollary 4.15).

0.7. Remark. The question of realizing homotopy actions is an old one, going back to work of Cooke in [C] (see also [LSm, O, Z, SV1]). Many approaches to this and related problems appear in the literature: since (homotopy) actions induce maps between classifying spaces of groups and monoids, any information about the latter is relevant to the question at hand. Methods for analyzing maps between classifying spaces were developed by Dwyer, Zabrodsky, Jackowski, McClure, Oliver, and others in the 1980's (cf. [DM, DZ, JMO]), and later by Grodal and Smith for actions on spheres, in [GS], based on Lannes theory (cf. [La]). Our approach here is more elementary, and perhaps more conceptual, although the machinery for calculating our obstructions is not as well-developed. 
0.8. Notation. The category of topological spaces will be denoted by $\mathcal{T}_{o p}$, and its objects will be denoted by boldface letters: $\mathbf{X}, \mathbf{Y} \ldots$ The category of pointed topological spaces $\mathbf{X}_{*}=\left(\mathbf{X}, x_{0}\right)$ is denoted by $\mathcal{T}_{o p_{*}}$.

A $G$-space is a topological space $\mathbf{X}$ equipped with a left $G$-action, and the category of $G$-spaces with $G$-maps (i.e., $G$-equivariant continuous maps) will be denoted by $G$-Top. We write $\mathbf{X}^{H}$ for the fixed point set $\{x \in \mathbf{X}: h x=x \forall h \in H\}$ of $X$ under a subgroup $H \leq G$.

An important example is a $G-C W$ complex, obtained by attaching $G$-cells of the form $G / H \times \mathbf{D}^{n+1}$ for $n \geq-1$ (see [I]). For finite $G$, this is equivalent to $\mathbf{X}$ being a CW-complex on which $G$ acts cellularly (see [tD, II, §1]).

An action of a (discrete) group $G$ on $\mathbf{X}$ is given by a homomorphism $\varphi_{\mathbf{X}}: G \rightarrow$ $\operatorname{Aut}(\mathbf{X})$, which we call the action map of $\mathbf{X}$. We call the composite $\zeta_{\mathbf{X}}:=i_{\mathbf{X}} \circ \varphi_{\mathbf{X}}$ : $G \rightarrow \operatorname{haut}(\mathbf{X})$ the monoid action map of $\mathbf{X}$, where $i_{\mathbf{X}}: \operatorname{Aut}(\mathbf{X}) \hookrightarrow \operatorname{haut}(\mathbf{X})$ is the inclusion. Similarly, a pointed action of $G$ on $\mathbf{X}_{*}$, given by the pointed action map $\varphi_{\mathbf{X}_{*}}^{*}: G \rightarrow \operatorname{Aut}_{*}\left(\mathbf{X}_{*}\right)$, has a pointed monoid action map $\zeta_{\mathbf{X}}^{*}:=i_{\mathbf{X}_{*}} \circ \varphi_{\mathbf{X}_{*}}^{*}: G \rightarrow$ $\operatorname{haut}_{*}\left(\mathbf{X}_{*}\right)$.

0.9. Organization. In Section 1 we provide some basic background on $G$-spaces, the orbit category, and equivariant homotopy theory. In Section 2 we address the question of transferring group actions along a map (cf. 0.5 ), as preparation for the interpolation problem, discussed in Section 3 (both for arbitrary groups). In Section 4 we define the notion of a Bredon homotopy action and prove our main result (for finite $G$ ). In the Appendix, we review the notion of a pointed homotopy action.

\section{G-Spaces and the Orbit Category}

In this section we recall some basic facts about $G$ spaces, and the Borel and Bredon approaches to equivariant homotopy theory.

1.1. Homotopy actions. Let haut $(\mathbf{X})$ denote the strictly associative topological monoid of self-homotopy equivalences of a topological space $\mathbf{X}$. If $G$ is a group, any monoid map $\zeta_{\mathbf{X}}: G \rightarrow$ haut $(\mathbf{X})$ factors through the submonoid $\operatorname{Aut}(\mathbf{X})$ of invertible elements (self-homeomorphisms) in haut(X), so it makes $\mathbf{X}$ into a $G$-space, equipped with a continuous $G$-action.

However, $\operatorname{Aut}(\mathbf{X})$ is not a homotopy invariant of $\mathbf{X}$, while haut $(\mathbf{X})$ is. A homotopy action of $G$ on $\mathbf{X}$ is therefore defined to be the homotopy class of a map $\Phi: \mathbf{B} G \rightarrow \mathbf{B}$ haut $(\mathbf{X})$ (see [DDK, DW] and compare [Su]). In particular, a group action determines a homotopy action, by setting $\Phi:=B \zeta_{\mathbf{X}}$.

If $\mathbf{X}_{*}=\left(\mathbf{X}, x_{0}\right)$ is pointed, haut $(\mathbf{X})$ has a sub-monoid haut $\left(\mathbf{X}_{*}\right)$ consisting of the pointed self-homotopy equivalences of $\mathbf{X}$, and a pointed homotopy action of $G$ on $\mathbf{X}_{*}$ is (the homotopy class of) a map $\Phi^{*}: \mathbf{B} G \rightarrow \mathbf{B}$ haut $_{*}\left(\mathbf{X}_{*}\right)$.

The inclusion $j: \operatorname{haut}_{*}\left(\mathbf{X}_{*}\right) \hookrightarrow$ haut $(\mathbf{X})$ fits into a homotopy fibration sequence:

$$
\operatorname{haut}_{*}\left(\mathbf{X}_{*}\right) \stackrel{j}{\rightarrow} \operatorname{haut}(\mathbf{X}) \stackrel{\mathrm{ev}^{x_{0}}}{\longrightarrow} \mathbf{X} \stackrel{k}{\rightarrow} \mathbf{B} \operatorname{haut}_{*}\left(\mathbf{X}_{*}\right) \stackrel{\mathbf{B} j}{\rightarrow} \mathbf{B} \operatorname{haut}(\mathbf{X})
$$

where $\mathbf{B} j$ is universal for Hurewicz fibrations with homotopy fiber $\mathbf{X}$ (cf. [A, $\mathbf{S t}$ ], and see [BGM, Theorem 5.6] \& [DFZ, Proposition 4.1]).

Note that a free $G$-space $\mathbf{X}$ is the total space of a principal $G$-bundle over the orbit space $\mathbf{X} / G$, which is classified by a map $\vartheta: \mathbf{X} / G \rightarrow \mathbf{B} G$. If we let 
$\mathbf{X}_{1} \hookrightarrow E_{\theta} \stackrel{\theta}{\rightarrow} \mathbf{B} G$ denote the pullback of $\mathbf{B} j$ along $\Phi$, this fits into a commuting diagram of fibration sequences:

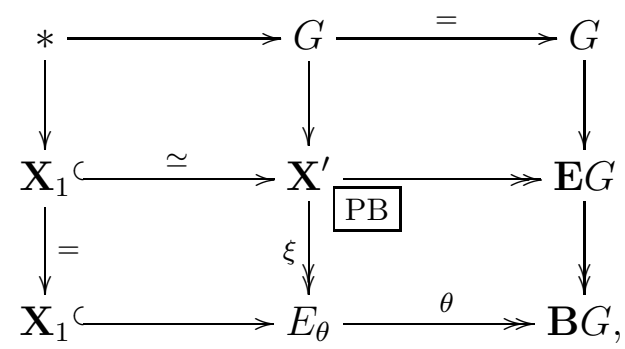

thus yielding a (free) topological $G$-action on $\mathbf{X}^{\prime} \sim \mathbf{X} \quad$ (with $\theta \in\left[\mathbf{X}^{\prime} / G, \mathbf{B} G\right]$ corresponding to $\vartheta \in[\mathbf{X} / G, \mathbf{B} G]$ under this equivalence, if $\mathbf{X}$ is a free $G$-space). Since we cannot guarantee that $G$ will act on $\mathbf{X}$ itself, this is sometimes refereed to as a proxy action (cf. [DW]).

Note that for every $G$-space $\mathbf{X}$ there is a $G$-map $\mathbf{X} \times \mathbf{E} G \rightarrow \mathbf{X}$ which is a homotopy equivalence (out of a free $G$-space). With this notion of $G$-weak equivalence, we obtain the Borel version of equivariant homotopy theory, which thus reduces to the study of principal $G$-bundles.

We say that the homotopy action $\Phi$ is realized by a free $G$-space $\mathbf{X}_{1}$ if the corresponding principal $G$-bundle (1.3) is classified by a map $\theta$ which is the pullback of (1.2) along $\Phi$; we have just seen that any homotopy action is realizable. Similarly, any pointed homotopy action is realizable by a pointed topological action (see Appendix).

Let $G$ be a fixed group. Bredon's approach to $G$-equivariant homotopy theory (cf. $[\mathrm{Br}, \mathrm{E}])$ reduces the study of a $G$-space $\mathbf{X}$ to the system of fixed point sets under the subgroups of $G$. To describe it, we recall the following:

1.4. The orbit category. The orbit category $\mathcal{O}_{G}$ of $G$ has the cosets $G / H$ (for each $H \leq G)$ as objects, and $G$-equivariant maps as morphisms.

Any map $G / H \rightarrow G / K$ in $\mathcal{O}_{G}$ can be factored as $i_{*}: G / H \rightarrow G / K^{a^{-1}}$ (induced by the inclusion $i: H \hookrightarrow K^{a^{-1}}$ ), followed by an isomorphism $\phi_{a}^{K^{a^{-1}}}$ : $G / K^{a^{-1}} \rightarrow G / K$, where $K^{a^{-1}}:=a K a^{-1}$, for $a \in G$, and $\phi_{a}^{K^{a^{-1}}}$ is induced by the right translation $g \mapsto a g$. Two maps $\phi_{a}^{K^{a^{-1}}} \circ i_{*}$ and $\phi_{b}^{K^{b^{-1}}} \circ j_{*}$ from $G / H$ to $G / K$ are the same in $\mathcal{O}_{G}$ if and only if $a^{-1} b \in K$. Thus the automorphism group $W_{H}:=\operatorname{Aut}_{\mathcal{O}_{G}}(G / H)$ of $G / H \in \mathcal{O}_{G}$ is $N_{G} H / H$ (where $N_{G} H$ is the normalizer of $H$ in $G$ ).

1.5. $\mathcal{O}_{G}$-diagrams. An $\mathcal{O}_{G}^{\mathrm{op}}$-diagram in $\mathcal{T} o p$ is a functor $\Psi: \mathcal{O}_{G}^{\mathrm{op}} \rightarrow \mathcal{T}$ op, and the category of all such will be denoted by $\mathcal{T}_{o p}{ }^{\mathcal{O}_{G}^{\circ}}$. The main example we have in mind is the fixed point set diagram $\underline{\mathrm{X}}$ associated a $G$-space $\mathbf{X}$, defined $\underline{\mathrm{X}}(G / H):=\mathbf{X}^{H}$.

Since $\mathcal{T}_{o p}$ is a simplicial model category, $\mathcal{T}_{o p}{ }^{\mathcal{O}_{G}^{\text {op }}}$ has a projective simplicial model category structure in which a map $f: \Psi \rightarrow \Psi^{\prime}$ of $\mathcal{O}_{G}^{\text {op }}$-diagrams is a weak equivalence (respectively, a fibration) if for each $H \leq G, f(G / H): \Psi(G / H) \rightarrow \Psi^{\prime}(G / H)$ is a weak equivalence (respectively, a fibration). See [Hi, Theorem 11.7.3].

There is an analogous simplicial model category structure on G-Top, in which a $G$-map $f: \mathbf{X} \rightarrow \mathbf{Y}$ is a weak equivalence (respectively, fibration) if for each 
$H \leq G$, the map $\left.f\right|_{\mathbf{X}^{H}}$ is a weak equivalence (respectively, fibration). See [DK1] and compare $[\mathrm{Pi}]$.

The following result of Elmendorf explains the central role of fixed-point sets in Bredon equivariant homotopy theory:

1.6. Theorem ([E, Theorem 1]). The fixed point set functor sending a $G$-space $\mathbf{X}$ to the diagram $\underline{X}: \mathcal{O}_{G}^{\text {op }} \rightarrow$ Top has a right adjoint $C: \mathcal{T}_{o p}{ }^{\mathcal{O}_{G}^{\text {op }}} \rightarrow G-\mathcal{T}$ op.

1.7. Remark. In fact, this adjoint pair constitutes a simplicial Quillen equivalence between $G$-Top and $\mathcal{T}_{o p}{ }^{\mathcal{O}_{G}^{o p}}$. Moreover, for any $G$-space X, Elmendorf shows that $C \underline{\mathrm{X}}$ is a $G$-CW complex. We therefore may (and shall) assume from now on that all our $G$-spaces are $G$-CW complexes.

1.8. Definition. A $G$-map $h: \mathbf{X} \rightarrow \mathbf{Y}$ which at the same time is a (non-equivariant) homotopy equivalence will be called a Borel $G$-equivalence. If $x_{0} \in \mathbf{X}$ and $y_{0}=$ $h\left(x_{0}\right) \in \mathbf{Y}$ are $G$-base-points (fixed under the $G$ action) and $h$ is a pointed homotopy equivalence, it will be called a pointed Borel G-equivalence.

1.9. Lemma. For any homotopy equivalence $h: \mathbf{X} \rightarrow \mathbf{Y}$ between $C W$ complexes, there is a $C W$ complex $\mathbf{Z}$ with homotopy equivalences $i: \mathbf{X} \rightarrow \mathbf{Z}$ and $i^{\prime}: \mathbf{Y} \rightarrow \mathbf{Z}$ such that $i \sim h \circ i^{\prime}$, inducing strictly multiplicative monic homotopy equivalences $i_{\star}: \operatorname{haut}(\mathbf{X}) \rightarrow \operatorname{haut}(\mathbf{Z})$ and $i_{\star}^{\prime}: \operatorname{haut}(\mathbf{Y}) \rightarrow \operatorname{haut}(\mathbf{Z})$.

Proof. Factoring $h$ as $p^{\prime} \circ i=h$ with $i$ a cofibration and $p^{\prime}$ a fibration, and using the cofibrancy of $\mathbf{X}$ and $\mathbf{Y}$, we obtain a diagram of homotopy equivalences

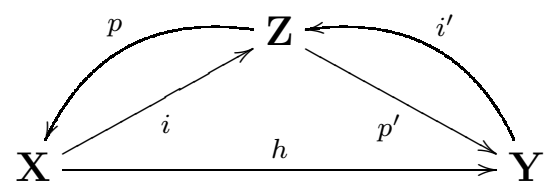

with $p \circ i=\operatorname{Id}_{\mathbf{X}}$ and $p^{\prime} \circ i^{\prime}=\operatorname{Id}_{\mathbf{Y}}$. Define $i_{\star}: \operatorname{haut}(\mathbf{X}) \rightarrow \operatorname{haut}(\mathbf{Z})$ by $\varphi \mapsto i \circ \varphi \circ p \quad$ (for any homotopy equivalence $\varphi: \mathbf{X} \rightarrow \mathbf{X}$ ). Because $p \circ i=\operatorname{Id}_{\mathbf{X}}$, the map $i_{\star}$ is monic, preserves compositions, and has a (non-monoidal) homotopy inverse $p_{*}$ : haut $(\mathbf{Z}) \rightarrow \operatorname{haut}(\mathbf{X})$. Similarly for $i^{\prime}$.

1.11. Remark. Any homotopy equivalence $h: \mathbf{X} \rightarrow \mathbf{Y}$ induces a homotopy equivalence $\mathbf{B}$ haut $(\mathbf{X}) \simeq \mathbf{B}$ haut $(\mathbf{Y}) \quad($ cf. [F1, Satz 7.7]). In fact, we can apply the classifying space functor $\mathbf{B}$ to the maps $i_{\star}$ and $i_{\star}^{\prime}$, obtaining homotopy equivalences:

$$
\mathbf{B} \text { haut }(\mathbf{X}) \stackrel{\mathbf{B} i_{\star}}{\longrightarrow} \mathbf{B} \text { haut }(\mathbf{Z}) \stackrel{\left(\mathbf{B} i_{\star}^{\prime}\right)^{-1}}{\longrightarrow} \mathbf{B} \operatorname{haut}(\mathbf{Y}),
$$

whose composite is denoted by $\mathbf{B} h_{*}$ (well-defined up to homotopy). Similarly in the pointed case.

\section{TRANSFERRING GROUP ACTIONS}

In this and the following section $G$ can be any topological group. Given a map $f: \mathbf{X} \rightarrow \mathbf{Y}$, consider the questions of:

- Transferring a given $G$-action on $\mathbf{X}$ along $f$ to $\mathbf{Y}$. or conversely.

- Making $f$ equivariant with respect to given actions on both $\mathbf{X}$ and $\mathbf{Y}$. 
In the spirit of [DK2], we shall show how they can be reduced to suitable lifting problems. First, we make the questions more precise:

2.1. Definition. Given any map $f: \mathbf{X} \rightarrow \mathbf{Y}$, a $G$-map $f^{\prime}: \mathbf{X}^{\prime} \rightarrow \mathbf{Y}^{\prime}$ is:

(i) a right transfer of a $G$-action on $\mathbf{X}$ along $f$ if we have a homotopy-commutative diagram

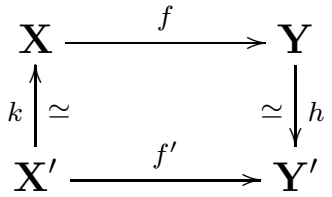

in which $h$ is a homotopy equivalence, and $k$ is a Borel $G$-equivalence.

(ii) a left transfer of a $G$-action on $\mathbf{Y}$ along $f$ if we have a diagram

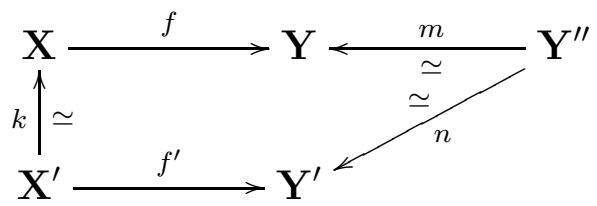

in which $k$ is a homotopy equivalence, $m$ and $n$ are Borel $G$-equivalences, which becomes homotopy-commutative after inverting $m$ or $n$ (up to homotopy).

(iii) a compatible $G$-map for $f$ with respect to $G$-actions on $\mathbf{X}$ and $\mathbf{Y}$ if we have a diagram

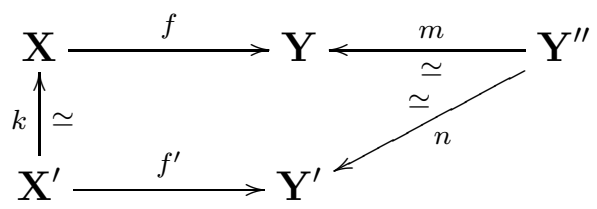

in which $k, m$, and $n$ are all Borel $G$-equivalences, which becomes homotopycommutative after inverting $m$ or $n$.

In order to describe the conditions under which such transfers exist, we require also the following construction:

2.5. Definition. For any map $f: \mathbf{X} \rightarrow \mathbf{Y}$, let $\mathcal{P}_{f}$ denote the homotopy pullback:

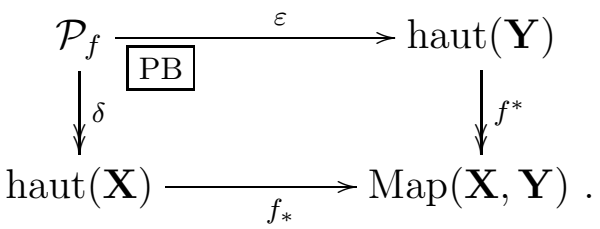

This can be constructed explicitly in two ways: if we change $f$ into a cofibration, the $\operatorname{map} f^{*}: \operatorname{Map}(\mathbf{Y}, \mathbf{Y}) \rightarrow \operatorname{Map}(\mathbf{X}, \mathbf{Y})$ is a fibration, so its restriction to $\operatorname{haut}(\mathbf{Y})$ is a fibration, too (since the latter is just a union of path components of $\operatorname{Map}(\mathbf{Y}, \mathbf{Y})$ ). In this case, the strict pullback is actually the homotopy pullback. Similarly when $f$ is a fibration, so $f_{*}$ is a fibration.

Using such a strict model, we see that $\mathcal{P}_{f}$ is a sub-monoid of the strictly associative monoid $\operatorname{haut}(\mathbf{X}) \times \operatorname{haut}(\mathbf{Y})$. Moreover, it is grouplike, since $(g, h) \in \mathcal{P}_{f}$ means that $f \circ g=h \circ f \quad\left(\right.$ for $g \in \operatorname{haut}(\mathbf{X})$ and $h \in \operatorname{haut}(\mathbf{Y})$ ), and thus $f \circ g^{-1} \sim h^{-1} \circ f$. 
If $f$ is either a fibration or a cofibration, we can use [BJT, Lemma 4.16] to change $h^{-1} \quad$ (respectively, $g^{-1}$ ) up to homotopy to get $f \circ g^{-1}=h^{-1} \circ f$ and thus $\left(g^{-1}, h^{-1}\right) \in \mathcal{P}_{f}$, too. The maps $\delta$ and $\varepsilon$ (the restrictions of the structure maps for $\check{\mathcal{P}}_{f}$ ) are monoid maps. Evidently $\mathcal{P}_{f}$ is a homotopy invariant of $f$.

With these notions we then have the following:

2.7. Proposition. Let $f: \mathbf{X} \rightarrow \mathbf{Y}$ be any map in $\mathcal{T}$ op.

(i) There is a right transfer of a $G$-action on $\mathbf{X}$ (with monoid action map $\zeta_{\mathbf{X}}$ : $G \rightarrow$ haut $(\mathbf{X})$ ) along $f$ if and only if there is a map $\Psi$ making the following diagram commute up to homotopy:

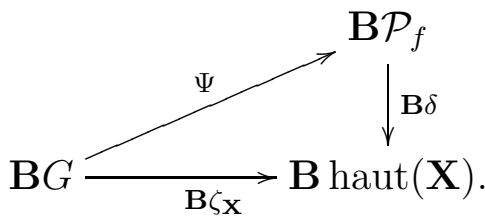

(ii) There is a left transfer of a $G$-action on $\mathbf{Y}$ (with monoid action map $\zeta_{\mathbf{Y}}$ : $G \rightarrow$ haut( $(\mathbf{Y})$ ) along $f$ if and only if there is a map $\Psi$ making the following diagram commute up to homotopy:

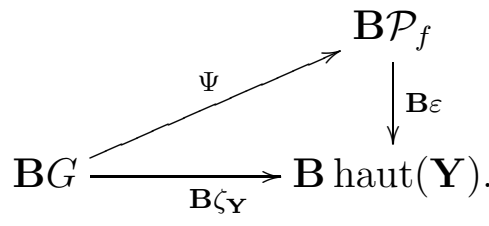

(iii) There is a compatible $G$-map for $f$ with respect to G-actions on $\mathbf{X}$ and $\mathbf{Y}$ (with monoid action maps $\zeta_{\mathbf{X}}: G \rightarrow \operatorname{haut}(\mathbf{X})$ and $\zeta_{\mathbf{Y}}: G \rightarrow \operatorname{haut}(\mathbf{Y})$ ) if and only if there is a map $\Psi$ making the following diagram commute up to homotopy:

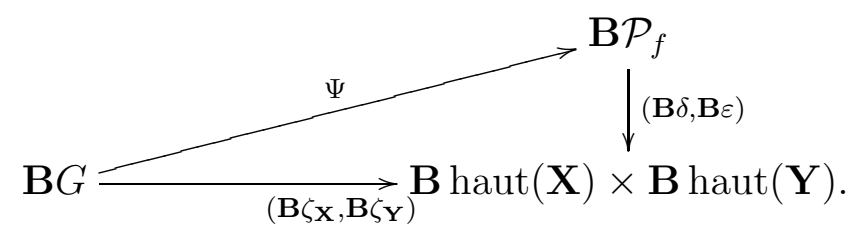

Proof. (i) If $\mathbf{X}$ is a $G$-space, and we have a right transfer $f^{\prime}: \mathbf{X}^{\prime} \rightarrow \mathbf{Y}^{\prime}$, of the $G$-action along $f$, we may change $f^{\prime}$ into a $G$-cofibration, and the monoid action maps $\zeta_{\mathbf{X}^{\prime}}: G \rightarrow \operatorname{haut}\left(\mathbf{X}^{\prime}\right)$ and $\zeta_{\mathbf{Y}^{\prime}}: G \rightarrow \operatorname{haut}\left(\mathbf{Y}^{\prime}\right)$ then fit together to define a monoid map $z: G \rightarrow \widetilde{\mathcal{P}}_{f^{\prime}}$ in (2.6), which actually lands in $\mathcal{P}_{f^{\prime}}$, since $G$ is a group. Because $\mathbf{B} \zeta_{\mathbf{x}}$ is just $\mathbf{B} \zeta_{\mathbf{X}^{\prime}}$, up to homotopy, $\mathbf{B} z: \mathbf{B} G \rightarrow \mathbf{B} \mathcal{P}_{f^{\prime}}$ is the required lift in (2.8).

Conversely, given a lift $\Psi$ in (2.8), by applying Kan's $G$-functor to (2.8), realizing, and then taking cofibrant replacement in the model category of strictly associative topological monoids (see [SV2, Theorem B]), we obtain a diagram of cofibrant (and 
grouplike) topological monoids:

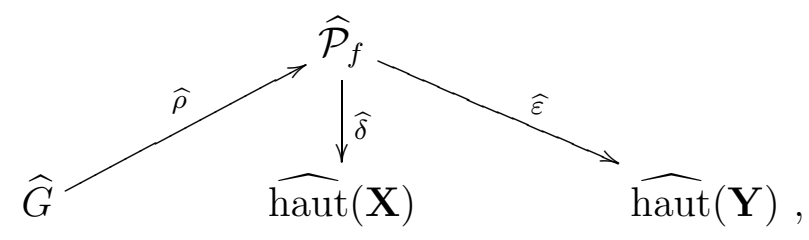

with $\widehat{\mathcal{P}}_{f}$ weakly equivalent to $\mathcal{P}_{f}$.

Since $\mathbf{B}$ haut $(\widehat{\mathbf{X}}) \simeq \mathbf{B}$ haut $(\mathbf{X})$, by pulling back (1.2) we obtain a monoid action of $\widehat{G}$ on $\widehat{\mathbf{X}} \simeq \mathbf{X}$. By [Pr, Theorem 5.8] (see also [DL, [F2], [Ma2, §7,9], [Bo, §5] and [DDK]), this is classified by a map $\hat{\theta}: E_{\hat{\theta}} \rightarrow \mathbf{B} \widehat{G}$. Up to homotopy, $\hat{\theta}$ corresponds to the map $\theta$ in the fibre bundle sequence:

$$
\mathbf{X}^{\prime}:=\mathbf{E} G \times \mathbf{X} \rightarrow E_{\theta} \stackrel{\theta}{\rightarrow} \mathbf{B} G
$$

classifying the free $G$-action on $\mathbf{X}^{\prime}$ (Borel equivalent to the given $\mathbf{X}$ ). Similarly, we get a free $\widehat{G}$-action on $\widehat{\mathbf{Y}} \simeq \mathbf{Y}$, classified by $\hat{\kappa}: E_{\hat{\kappa}} \rightarrow \mathbf{B} \widehat{G}$ Moreover, we have a map $\widehat{f}: \widehat{\mathbf{X}} \rightarrow \widehat{\mathbf{Y}}$ (which is just $f: X \rightarrow Y$, up to homotopy), and we may assume that $\widehat{f}$ is itself a cofibration (for example, by carrying out the above construction in simplicial sets, and replacing $\widehat{\mathbf{Y}}$ by $\widehat{\mathbf{Y}} \times \mathbf{C} \widehat{\mathbf{X}}$ before realizing).

As in (2.6), we obtain a commuting diagram

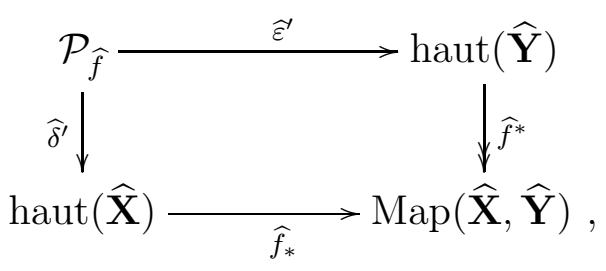

in which $\widehat{f}^{*}$ is a fibration, so $\widehat{\delta}^{\prime}$ is, too.

Because $\widehat{\mathbf{X}} \simeq \mathbf{X}, \widehat{\operatorname{haut}}(\mathbf{X})$ and $\operatorname{haut}(\mathbf{X})$ are weakly equivalent, and since the former is cofibrant and the latter is fibrant, we have a weak equivalence of monoids $k: \widehat{\operatorname{haut}}(\mathbf{X}) \rightarrow \operatorname{haut}(\mathbf{X})$, and similarly $\ell: \widehat{\operatorname{haut}}(\mathbf{Y}) \simeq \operatorname{haut}(\mathbf{Y})$. Moreover, since (2.13) is a homotopy pullback, $\mathcal{P}_{f}$ and $\mathcal{P}_{\widehat{f}}$ are weakly equivalent, and again we have a weak equivalence of monoids $h: \widehat{\mathcal{P}}_{f} \stackrel{\widetilde{\sim}}{\rightarrow} \mathcal{P}_{\widehat{f}}$. Thus the strict diagram (2.13) fits into a homotopy commutative diagram:

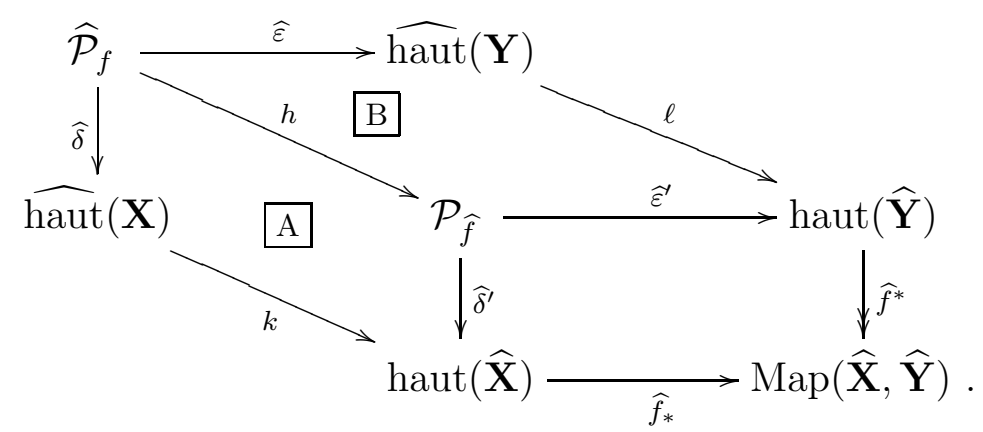

In the model category of strictly associative monoids, we can replace $h$ by another weak equivalence of monoids making $\mathrm{A}$ commute on the nose (cf. [BJT, Lemma 
4.16]), and then changing $\ell$ into a fibration, we may replace $\widehat{\varepsilon}$ by a map making $B$ commute strictly, too, without changing $\widehat{\mathcal{P}}_{f}$.

Composing the monoid map $\widehat{\rho}: \widehat{G} \rightarrow \widehat{\mathcal{P}}_{f}$ of (2.11) with $k \circ \widehat{\delta}: \widehat{\mathcal{P}}_{f} \rightarrow \operatorname{haut}(\widehat{\mathbf{X}})$ and $\ell \circ \widehat{\varepsilon}: \widehat{\mathcal{P}}_{f} \rightarrow \operatorname{haut}(\widehat{\mathbf{Y}})$, we obtain monoid action maps $\widehat{\zeta}_{\widehat{\mathbf{X}}}: \widehat{G} \rightarrow \operatorname{haut}(\widehat{\mathbf{X}})$ and $\widehat{\zeta}_{\widehat{\mathbf{Y}}}: \widehat{G} \rightarrow \operatorname{haut}(\widehat{\mathbf{Y}})$ making $\widehat{\mathbf{X}}$ and $\widehat{\mathbf{Y}}$ into strict $\widehat{G}$-spaces, with $\widehat{f}: \widehat{\mathbf{X}} \hookrightarrow \widehat{\mathbf{Y}} \quad$ a $\widehat{G}$ map (which is a cofibration). It therefore fits into a commuting diagram of principal $\widehat{G}$-bundles

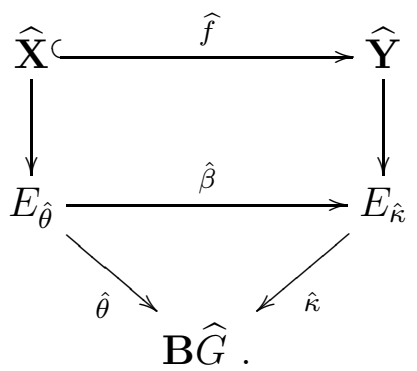

Here $\hat{\beta}$ is obtained by realizing the bar construction for the $\widehat{G}$-actions on $\widehat{\mathbf{X}}$ and $\widehat{\mathbf{Y}}$, respectively (see $[\mathrm{Pr}, \S 5]$ ), so it commutes up to homotopy with the classifying maps $\hat{\theta}$ and $\hat{\kappa}$ for the two bundles, where (as noted above) up to homotopy $\hat{\theta}$ is just $\theta: E_{\theta} \rightarrow \mathbf{B} G$, classifying the free $G$-space $\mathbf{X}^{\prime}$.

Let $\kappa: E_{\hat{\kappa}} \rightarrow \mathbf{B} G$ denote the composite of $\hat{\kappa}$ with $\mathbf{B} \widehat{G} \simeq \mathbf{B} G$, classifying a free $G$-bundle $\mathbf{Y}^{\prime} \rightarrow E_{\hat{\kappa}}$ with $\mathbf{Y}^{\prime} \simeq \widehat{\mathbf{Y}} \simeq \mathbf{Y}$. If we also let $\beta: E_{\theta} \rightarrow E_{\hat{\kappa}}$ denote the composite of $\hat{\beta}$ with $E_{\theta} \simeq E_{\hat{\theta}}$, then $\kappa \circ \beta \simeq \theta$, so we have a map

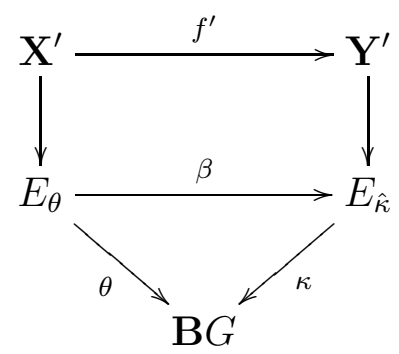

of principal $G$-bundles, so in particular, $f^{\prime}$ is a $G$-map.

Statements (ii) and (iii) are proven analogously.

Compare [Z, Proposition 2.2] for the compatibility version for homotopy actions.

2.17. Proposition. Let $f: \mathbf{X} \rightarrow \mathbf{Y}$ be any map. In a right transfer of a G-action on $\mathbf{X}$ along $f$, we may assume that $k$ in (2.2) is a homeomorphism; in a left transfer of a $G$-action on $\mathbf{Y}$ along $f$, we may assume that $m$ and $n$ in (2.3) are homeomorphisms; and in a compatible $G$-map for $f$ with respect to $G$-actions on $\mathbf{X}$ and $\mathbf{Y}$, we may assume that either $k$, or $m$ and $n$, are homeomorphisms in 2.4).

Proof. If the action of $G$ on $\mathbf{X}$ is free (and (2.8) holds), we can replace $\mathbf{X}^{\prime}:=\mathbf{E} G \times \mathbf{X}$ by $\mathbf{X}$ in (2.12), and therefore also in (2.16), so we have a right transfer $f^{\prime}: \mathbf{X} \hookrightarrow \mathbf{Y}^{\prime}$ (along $f: \mathbf{X} \rightarrow \mathbf{Y}$ ) which is a $G$-map.

The same argument shows that if the action of $G$ on $\mathbf{Y}$ is free (and (2.9) holds), it has a left transfer along $f$ to a fibration $f^{\prime}: \mathbf{X}^{\prime} \rightarrow \mathbf{Y}$ which is a $G$-map. Moreover, 
given free $G$-actions on both $\mathbf{X}$ and $\mathbf{Y}$, any $f: \mathbf{X} \rightarrow \mathbf{Y}$ has a compatible $G$-map $f^{\prime}: \mathbf{X} \rightarrow \mathbf{Y}$ with the same source and target (if (2.10) holds).

Since every $G$-space $\mathbf{Y}$ has a Borel $G$-equivalence $h: \mathbf{Y}^{\prime} \rightarrow \mathbf{Y}$, where $\mathbf{Y}^{\prime}$ is a free $G$-space, we do not actually need to assume that the action on $\mathbf{Y}$ is free, because we can compose the left transfer or compatible map $f^{\prime}$ with this $h$. Thus we may always assume that $\ell, m$, and $n$ are homeomorphisms.

Finally, given a $G$-space $\mathbf{X}$, we may replace it by the free $G$-space $\mathbf{X}^{\prime}:=\mathbf{X} \times \mathbf{E} G$ and produce a $G$-map $f^{\prime}: \mathbf{X}^{\prime} \hookrightarrow \mathbf{Y}^{\prime}$ which is a cofibration. Then taking the pushout:

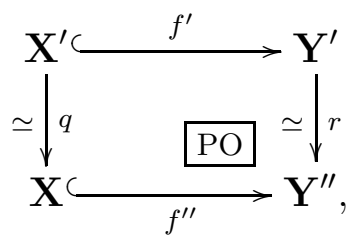

we see that all maps are $G$-maps; $f^{\prime}$, and thus $f^{\prime \prime}$, are cofibrations, so this is a homotopy pushout in $\mathcal{T} o p$, and since $q$ is a homotopy equivalences, so is $r$.

2.19. Applications. In general, the lifting problems of Proposition 2.7 are hard to solve. However, in certain cases the obstructions to obtain the relevant liftings may be computable, or may vanish for dimension reasons. For example:

(i) When $\mathbf{X}=K(\pi, n)$ is an Eilenberg-Mac Lane space, then:

$$
\operatorname{haut}(\mathbf{X}) \simeq K(\pi, n) \times \operatorname{Aut}(\pi)
$$

(as a monoid) is a semi-direct product of the Eilenberg-Mac Lane space itself and the discrete group $\operatorname{Aut}(\pi)$, while $\operatorname{haut}_{*}(X) \simeq \operatorname{Aut}(\pi) \cong \pi_{0} \operatorname{haut}_{*}(X)$ is homotopically discrete (see [Ma1, Proposition 25.2]).

Thus if both $\mathbf{X}$ and $\mathbf{Y}$ are Eilenberg-Mac Lane spaces, all but the pullback itself in (2.6) are generalized Eilenberg-Mac Lane spaces, so each of the lifting problems (2.8), (2.9), and (2.10) reduces to an algebraic question about certain classes in the cohomology of $\mathbf{B} G$ (as expected).

(ii) A more interesting example is when each of $\mathbf{X}$ and $\mathbf{Y}$ has only two non-trivial homotopy groups (see 3.16 below). In this case the homotopy groups of haut(X) and haut(Y) are completely known by [Di, §3] (see also [Mo]), and in particular if $\pi_{i} \mathbf{X}=0$ for $i \neq k, m \quad(k<m)$, then $\pi_{i} \operatorname{haut}(\mathbf{X})=0$ unless $k \leq i \leq m$. Using the Postnikov tower for $\mathbf{Y}$ we can also determine $\pi_{*} \operatorname{Map}(\mathbf{X}, \mathbf{Y})$, up to extension. Therefore the homotopy groups of $\mathcal{P}_{f}$ may also be determined, up to extension.

Since we need not assume $G$ is finite, $H^{i}(\mathbf{B} G ; \pi)$ may vanish for large enough $i$, at least when $\pi$ is one of the groups $\pi_{*} \mathcal{P}_{f} \quad$ Thus in certain cases we can show that there is no obstruction to solving the lifting problems.

(iii) The case when $\mathbf{X}$ and $\mathbf{Y}$ are spheres has been the subject of intense study over the years, beginning with [PSm]. Moreover, much is known about haut $\left(\mathbf{S}^{n}\right)$ and $\operatorname{haut}_{*}\left(\mathbf{S}^{n}\right)$ (see, e.g., [Ha1, Ha2]). Therefore, one might be able to compute obstructions to extending or lifting certain group actions on spheres along some map $f: \mathbf{S}^{n} \rightarrow \mathbf{S}^{m}$, or making $f$ equivariant. 


\section{INTERPOLATING GROUP ACTIONS}

For our approach to the realization problem for Bredon homotopy actions, we need to consider a slightly more complicated situation than that studied in the previous section: assume given a sequence of maps

$$
\mathbf{X} \stackrel{f}{\rightarrow} \mathbf{Y} \stackrel{g}{\rightarrow} \mathbf{Z}
$$

with given $G$-actions on $\mathbf{X}$ and $\mathbf{Z}$, for which we want to find a compatible $G$-action on $\mathbf{Y}$.

3.2. Definition. A $G$-interpolation for two $G$-spaces $\mathbf{X}$ and $\mathbf{Z}$ and maps as in (3.1) is a pair of $G$-maps $f^{\prime}: \mathbf{X}^{\prime} \rightarrow \mathbf{Y}^{\prime}$ and $g^{\prime}: \mathbf{Y}^{\prime} \rightarrow \mathbf{Z}^{\prime}$ fitting into a diagram

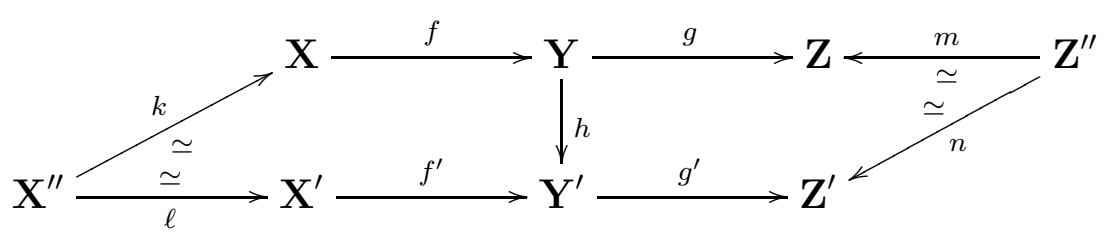

in which $k, \ell, m$, and $n$ are all homotopy equivalences and $G$-maps, and $h$ is a homotopy equivalence, which becomes homotopy-commutative after inverting $m$ or $n$ (up to homotopy).

3.4. Definition. Given two composable maps as in (3.1), let $\mathcal{Q}_{f, g}$ denote the homotopy pullback in:

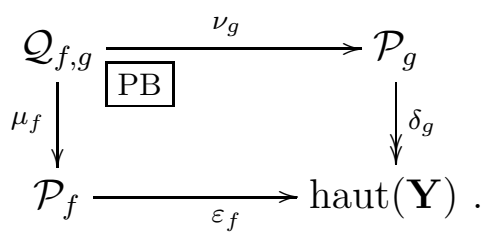

(see 2.5). If $f$ and $g$ are cofibrations, both $\mathcal{P}_{f}$ and $\mathcal{P}_{g}$ are actually pullbacks, and the map $\delta: \mathcal{P}_{g} \rightarrow$ haut $(\mathbf{Y})$ is a fibration, so $\mathcal{Q}_{f, g}$ is the ordinary pullback. Furthermore, it is a grouplike strictly associative monoid, and the maps $\mu$ and $\nu$ are monoid maps.

3.6. Proposition. Two maps as in (3.1) for $G$-spaces $\mathbf{X}$ and $\mathbf{Z}$ (with monoid action maps $\zeta_{\mathbf{X}}: G \rightarrow$ haut $(\mathbf{X})$ and $\zeta_{\mathbf{Z}}: G \rightarrow$ haut $(\mathbf{Z})$, respectively) have a $G$ interpolation if and only if there is a map $\Psi$ making the following diagram commute up to homotopy:

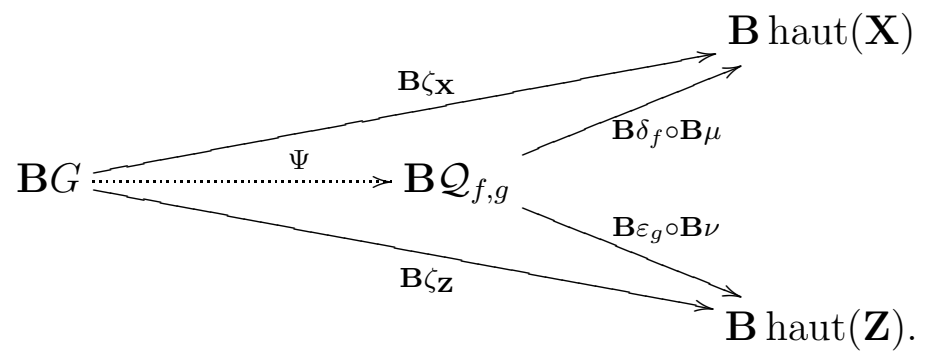

Proof. Given a $G$-interpolation, the diagram (3.7) is obtained by applying B to the corresponding monoid action maps. 
Conversely, a homotopy commutative diagram (3.7) may be lifted (together with (3.5)) to a commuting diagram of topological monoids:

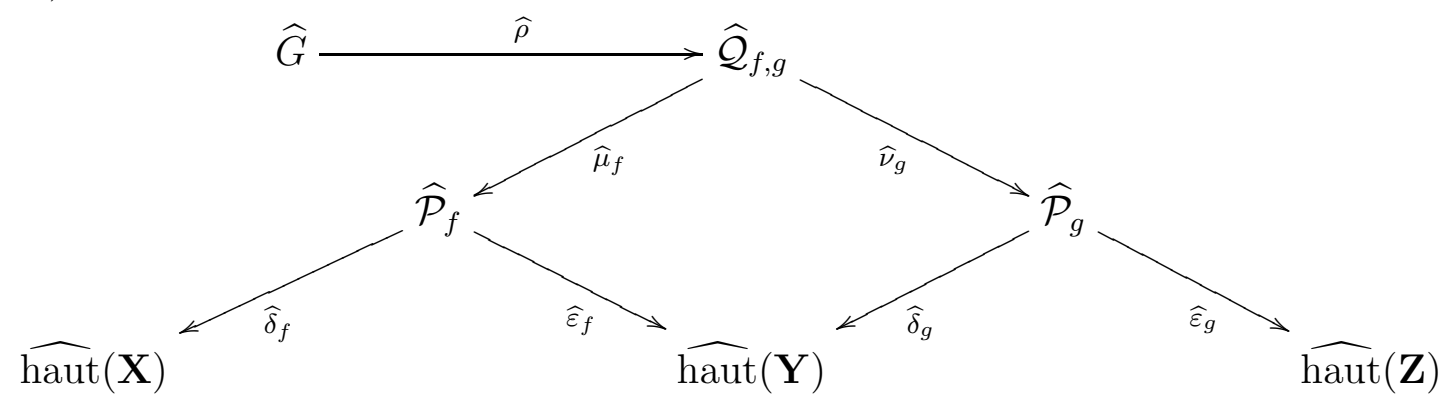

and we have spaces $\widehat{\mathbf{X}} \simeq \mathbf{X}, \quad \widehat{\mathbf{Y}} \simeq \mathbf{Y}$, and $\widehat{\mathbf{Z}} \simeq \mathbf{Z}$ on which $\widehat{\operatorname{haut}}(\mathbf{X})$, $\widehat{\operatorname{haut}}(\mathbf{Y})$, and $\widehat{\operatorname{haut}}(\mathbf{Z})$, respectively act. Moreover, we have maps $\widehat{f}: \widehat{\mathbf{X}} \rightarrow \widehat{\mathbf{Y}}$ and $\widehat{g}: \widehat{\mathbf{Y}} \rightarrow \widehat{\mathbf{Z}} \quad$ (corresponding up to homotopy to $f$ and $g$, respectively), and as in the proof of Proposition [2.7, we may assume $\widehat{f}$ and $\widehat{g}$ are cofibrations.

Therefore, (3.8) fits into a diagram:

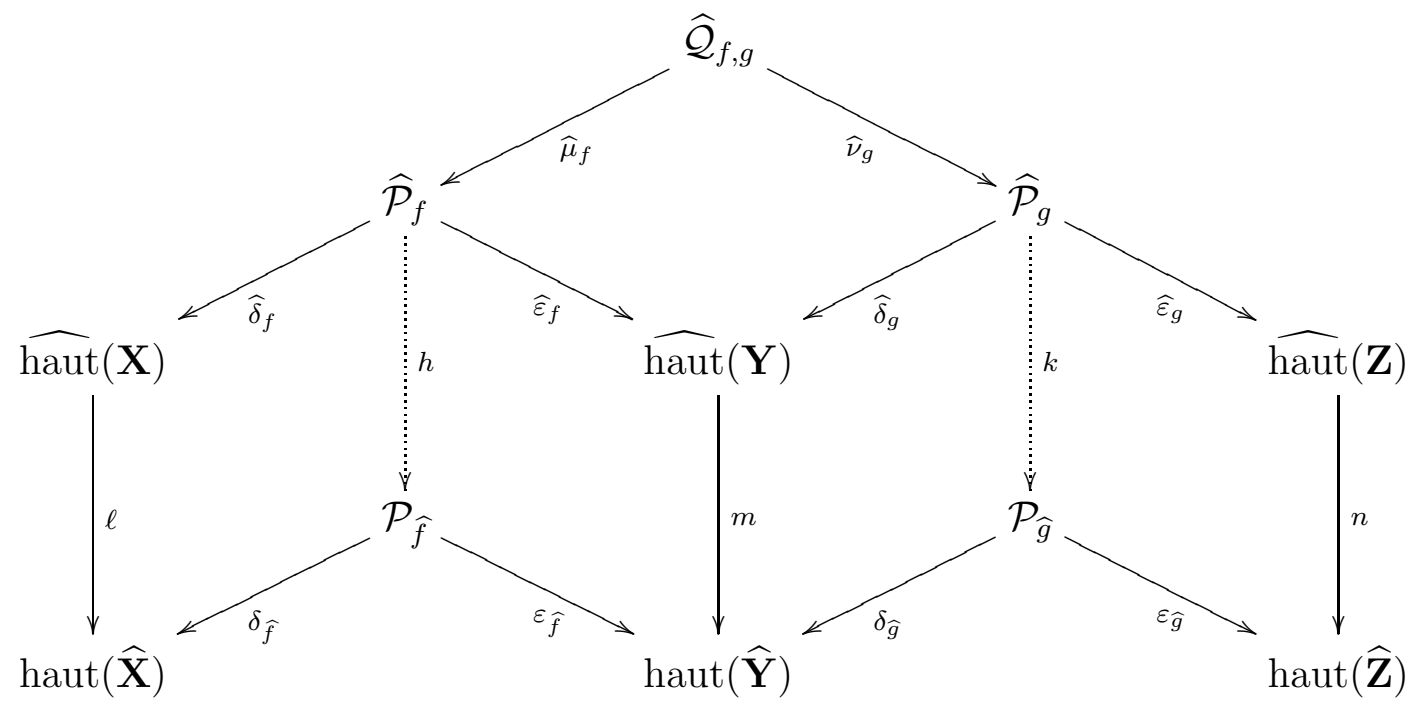

in which $\mathcal{P}_{\widehat{f}}$ and $\mathcal{P}_{\widehat{g}}$ are homotopy limits, so the homotopy equivalences $\ell, m$, and $n$ induce homotopy equivalences $h$ and $k$ making (3.9) homotopy-commutative. Since $\mathcal{Q}_{\widehat{f}, \widehat{g}}$ is also a homotopy limit, $h$ and $k$ induce a homotopy equivalence $p: \widehat{\mathcal{Q}}_{f, g} \rightarrow \mathcal{Q}_{\widehat{f}, \widehat{g}}$.

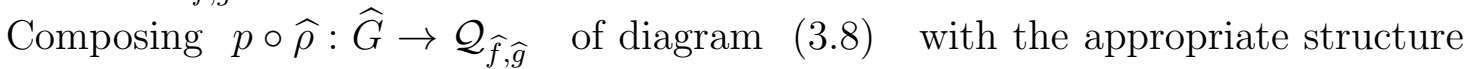
maps for $\mathcal{Q}_{\widehat{f}, \widehat{g}}$ yields monoid action maps $\widehat{\zeta}_{\widehat{\mathbf{X}}}: \widehat{G} \rightarrow \operatorname{haut}(\widehat{\mathbf{X}}), \quad \widehat{\zeta}_{\widehat{\mathbf{Y}}}: \widehat{G} \rightarrow \operatorname{haut}(\widehat{\mathbf{Y}})$, and $\widehat{\zeta}_{\widehat{\mathbf{Z}}}: \widehat{G} \rightarrow \operatorname{haut}(\widehat{\mathbf{Z}})$ making $\widehat{f}$ and $\widehat{g}$ into $\widehat{G}$-equivariant maps (by definition of $\left.\mathcal{Q}_{\widehat{f}, \widehat{g}}\right)$, with $\widehat{\zeta}_{\widehat{\mathbf{X}}}$ and $\widehat{\zeta}_{\widehat{\mathbf{Z}}}$ corresponding up to homotopy to the given monoid action maps $\zeta_{\mathbf{X}}: G \rightarrow \operatorname{haut}(\mathbf{X})$ and $\zeta_{\mathbf{Z}}: G \rightarrow \operatorname{haut}(\mathbf{Z})$. 
Passing to the classifying maps for the corresponding principle $G$ - and $\widehat{G}$-bundles as in the proof of Proposition 2.7, we obtain the required $G$-interpolation.

We now have the following analogue of Proposition 2.17;

3.10. Proposition. If $p:=g \circ f: \mathbf{X} \rightarrow \mathbf{Z}$ is a $G$-map in (3.1), in any $G$ interpolation for we may assume that $k, \ell, m$, and $n$ are homeomorphisms in (3.3), and that $g^{\prime} \circ f^{\prime}=p$.

Proof. In the proof of Proposition 3.6 we saw that the lifting $\Psi$ in (3.7) allows us to factor the map of free $G$-spaces (i.e., total spaces of principle $G$-bundles)

$$
\mathbf{X}^{\prime}:=\mathbf{X} \times \mathbf{E} G \stackrel{p^{\prime}:=p \times \operatorname{Id}_{\mathbf{E} G}}{\longrightarrow} \mathbf{Z} \times \mathbf{E} G=: \mathbf{Z}^{\prime}
$$

as the composite of two maps of free $G$-spaces $\mathbf{X}^{\prime} \stackrel{f^{\prime}}{\rightarrow} \mathbf{Y}^{\prime} \stackrel{g^{\prime}}{\rightarrow} \mathbf{Z}^{\prime}$ with $\mathbf{Y} \simeq \mathbf{Y}^{\prime}$ (using a homotopy-factorization of the corresponding classifying maps of the bundles). Applying the (homotopy) pushout (2.18) we obtain a commutative diagram of $G$ spaces:

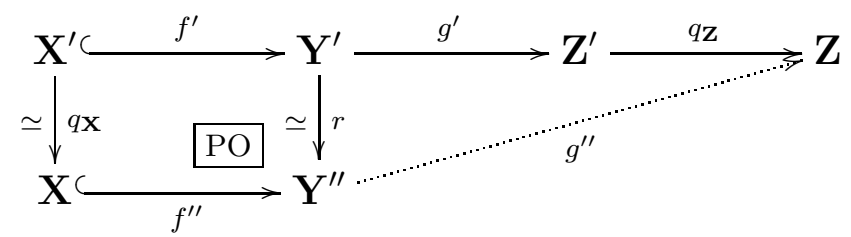

where the map $g^{\prime \prime} \quad$ out of the pushout is induced by $q_{\mathbf{Z}} \circ g^{\prime}: \mathbf{Y}^{\prime} \rightarrow \mathbf{Z}$ and $p:=f \circ g: \mathbf{X} \rightarrow \mathbf{Z}$, which agree on $\mathbf{X}^{\prime}$ since $p^{\prime}=g^{\prime} \circ f^{\prime}=(g \circ f) \times \operatorname{Id}_{\mathbf{E} G}$.

3.12. Definition. Given two maps as in (3.1) for $G$-spaces $\mathbf{X}$ and $\mathbf{Z}$, let $\rho$ : $\mathbf{B} \mathcal{Q}_{f, g} \rightarrow \mathbf{B}$ haut $(\mathbf{X}) \times \mathbf{B}$ haut $(\mathbf{Z})$ be the map $\left(\mathbf{B} \delta_{f} \circ \mathbf{B} \mu, \mathbf{B} \varepsilon_{g} \circ \mathbf{B} \nu\right)$ of (3.7), and let:

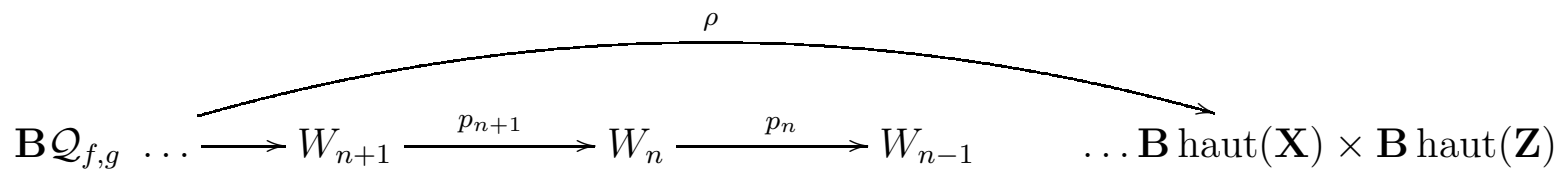

be the Moore-Postnikov tower for $\rho$, with $W_{0}:=\mathbf{B}$ haut $(\mathbf{X}) \times \mathbf{B}$ haut $(\mathbf{Z}) \quad$ (cf. GJ, VI, 3.9]).

If $F$ denotes the homotopy fiber of $\rho$, then up to homotopy each map $p_{n+1}$ : $W_{n+1} \rightarrow W_{n}$ is a fibration with fiber $K\left(\pi_{n+1} F, n+1\right)$, which is classified by a map $\tilde{k}_{n}: W_{n} \rightarrow K_{\pi_{1} Q_{n}}\left(\pi_{n+1} F, n+2\right) \quad$ (see [R, Theorem 3.4]). Assume by induction on $n \geq 0$ that we have constructed a lift $g_{n}: \mathbf{B} G \rightarrow W_{n}$ for $g_{0}:=\left(\mathbf{B} \zeta_{\mathbf{X}}, \mathbf{B} \zeta_{\mathbf{z}}\right)$ : $\mathbf{B} G \rightarrow W_{0}=\mathbf{B}$ haut $(\mathbf{X}) \times \mathbf{B}$ haut $(\mathbf{Z})$. Then the $n$-th obstruction class for this lift is

$$
\left[\tilde{k}_{n} \circ g_{n}\right] \in\left[\mathbf{B} G, K_{\pi_{1} Q_{n}}\left(\pi_{n+1} F, n+2\right)\right] \cong H^{n+2}\left(G ; \pi_{n+1} F\right)
$$

where $G$ acts on $\pi_{n+1} F$ via $\left(g_{n}\right)_{\#}: G \rightarrow \pi_{1} W_{n}$.

From Proposition 3.6 we deduce:

3.14. Proposition. Two maps as in (3.1) for $G$-spaces $\mathbf{X}$ and $\mathbf{Z}$ (with monoid action maps $\zeta_{\mathbf{X}}: G \rightarrow$ haut $(\mathbf{X})$ and $\zeta_{\mathbf{Z}}: G \rightarrow$ haut $(\mathbf{Z})$, respectively) have a $G$-interpolation if and only if the obstruction classes $\left[\tilde{k}_{n} \circ g_{n}\right] \in H^{n+2}\left(G ; \pi_{n+1} F\right)$ 
successively vanish, for some sequence of lifts. There is also a sequence of difference obstructions in $H^{n+1}\left(G ; \pi_{n+1} F\right)$ for distinguishing between non-homotopic lifts in (3.7).

3.15. Remark. As with any obstruction theory, non-vanishing of a cohomology class $\left[\tilde{k}_{n} \circ g_{n}\right]$ merely requires that we back-track to an earlier stage and try different choices, so in reality we have tree of obstructions, and the $G$-interpolation exists if and only if some branch extends to infinity.

3.16. Example. We can use the method described here to study $G$-actions on a space $\mathbf{Y}$ if $\pi_{i} \mathbf{Y}=0$ for $i \neq k, m \quad(k<m)$ : In this case we can choose in $\mathbf{X}:=K(\pi, m)$ and $\mathbf{Z}:=K\left(\pi^{\prime}, k\right)$ in (3.1), with given actions of $G$ on $\pi$ and $\pi^{\prime}$, and use Proposition 3.6 to interpolate a $G$-action on Y. As noted in $\$ 2.19$, for suitable choices of $G$ the obstructions of Proposition 3.14 will vanish (e.g., for reasons of dimension).

\section{REALizing DiAGRAMMATiC HOMOTOPY ACTIONS}

From now on we assume that $G$ is finite (but see 4.16 below). Given a $G$-space $\mathbf{X}$, the associated fixed point set diagram $\underline{\mathrm{X}}$ encodes the Bredon $G$-homotopy type of $\mathbf{X}$, by Theorem [1.6. This diagram consists of the various fixed point sets $\mathbf{X}^{H}$ $(H \leq G)$, the inclusions $i^{*}: \mathbf{X}^{K} \hookrightarrow \mathbf{X}^{H}$ induced by $i: H \hookrightarrow K$, and the $G$-action by conjugation: $\mathbf{X}^{H} \rightarrow \mathbf{X}^{H^{a}}$. Our goal is to provide a "homotopy version" of $\underline{\mathbf{X}}$, and describe a procedure for realizing it by attempting to solve a sequence of simpler lifting problems as in Section 3 ,

4.1. Filtering $\mathcal{O}_{G}^{\text {op }}$. For any subgroup $H$ of $G$, we define the length of $H$ in $G$, denoted by $\operatorname{len}_{G} H$, to be the maximal $0 \leq k<\infty$ such that there exists a sequence of proper inclusions of subgroups:

$$
H=H_{0}<H_{1}<H_{2}<\ldots<H_{k-1}<H_{k}=G .
$$

This induces a filtration

$$
\mathcal{F}_{0} \subset \mathcal{F}_{1} \subset \ldots \mathcal{F}_{k} \subset \ldots \subset \mathcal{O}_{G}^{\text {op }}
$$

by full subcategories, where $\operatorname{Obj} \mathcal{F}_{k}:=\left\{G / H \in \mathcal{O}_{G}^{\text {op }}: \operatorname{len}_{G} H \leq k\right\} \quad$ (so Obj $\mathcal{F}_{0}=$ $\{G / G\})$.

Since $G$ is finite, the filtration is exhaustive: if $\operatorname{len}_{G}\{e\}=N$ - that is, the longest possible sequence (4.2) in $G$ has $N$ inclusions of proper subgroups - then $\mathcal{F}_{N}=\mathcal{O}_{G}^{\text {op }}$. We let $\widehat{\mathcal{F}}_{k}$ denote the collection of subgroups $H<G$ such that $G / H \in \mathcal{F}_{k}$.

Let $\langle H\rangle:=\left\{H^{a}: a \in G\right\}$ denote the conjugacy class of a subgroup $H \leq G$ : note that if $H \in \widehat{\mathcal{F}}_{k}$, then $\langle H\rangle \subseteq \widehat{\mathcal{F}}_{k}$.

4.4. Definition. Let $\Lambda$ denote the partially ordered set of subgroups of $G$; we can think of the opposite category $\Lambda^{\mathrm{op}}$ as a subcategory of $\mathcal{O}_{G}$. The full subcategory $\Lambda_{H}$ consists of all subgroups $K$ with $H<K \leq G$, and $\Lambda_{k}$ is the full subcategory of objects in filtration $\mathcal{F}_{k}$.

4.5. Definition. A Bredon homotopy action of $\left.G \underset{\sim}{\sim},\left(\Phi_{H}^{*}\right)_{\langle H\rangle \subseteq \Lambda}\right\rangle$ consists of: 
(i) A diagram $\underset{\sim}{X}: \Lambda^{\mathrm{op}} \rightarrow$ Top.

(ii) A choice of a representative $H$ in each conjugacy class $\langle H\rangle \subseteq \Lambda$, equipped with a pointed homotopy $W_{H}$-action $\Phi_{H}^{*}: \mathbf{B} W_{H} \rightarrow \mathbf{B}$ haut $_{*}\left(\underset{\sim}{\mathbf{X}_{H}^{H}}\right) \quad$ on $\underset{\sim}{\mathbf{X}_{H}^{H}}$, defined by the homotopy cofibration sequence:

$$
\underset{\sim}{\mathbf{X}} \rightarrow \underset{\sim}{X}(H) \rightarrow \underset{\sim}{\mathbf{X}_{H}^{H}}
$$

where $\underset{\sim}{\mathbf{X}}:=$ hocolim $_{\Lambda_{H}^{\text {op }}} \underset{\sim}{X}(K)$.

We require that if $H^{\prime}$ and $H$ are conjugate, their homotopy cofibration sequences (4.6) fit into a homotopy-commuting square (0.2).

4.7. Definition. A cofibrant diagram $\underline{\mathrm{X}}_{k}: \mathcal{F}_{k} \rightarrow \mathcal{T}$ op (in the projective model category $\mathcal{T}_{o p} \mathcal{F}_{k}$ - cf. [Hi, §11.6]) realizes a Bredon homotopy action $\left.\underset{\sim}{\sim},\left(\Phi_{H}^{*}\right)_{H \leq G}\right\rangle$ in the $k$-th filtration if:

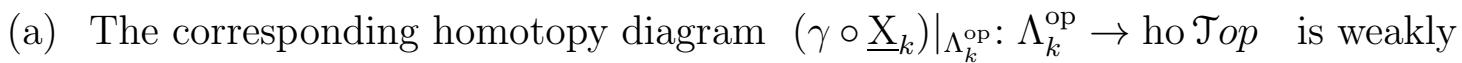
equivalent to $\left.\gamma \circ \underset{\sim}{X}\right|_{\Lambda_{k}^{\text {op }}}$, for $\gamma: \mathcal{T} o p \rightarrow$ ho $\mathcal{T}_{o p}$ the quotient functor.

(b) For each $H \in \mathcal{F}_{k}$, the pointed action of $W_{H}$ on the cofiber of

$$
\operatorname{colim}_{K>H} \underline{\mathrm{X}}_{k}(G / K) \rightarrow \underline{\mathrm{X}}_{k}(G / H)
$$

realizes the pointed homotopy action $\Phi_{H}^{*}$.

Note that because $\underline{X}_{k}$ is cofibrant, this colimit is a homotopy colimit and (4.8) is a cofibration, and because of (0.2), the homotopy action $\Phi_{H}^{*}$ is defined for every $H \leq G$, not only our chosen representatives.

A sequence $\left(\underline{\mathrm{X}}_{k}: \mathcal{F}_{k} \rightarrow \mathcal{T} o p\right)_{k=0}^{\infty}$ of such diagrams is coherent if $\left.\underline{\mathrm{X}}_{k}\right|_{\mathcal{F}_{k-1}}=\underline{\mathrm{X}}_{k-1}$ for each $k \geq 1$.

4.9. Example. If $\mathbf{X}$ is a $G$-CW complex, let $\underset{\sim}{X}$ be the restriction of $\underline{\mathrm{X}}: \mathcal{O}_{G}^{\text {op }} \rightarrow \mathcal{T}$ op to the subcategory $\Lambda^{\text {op }}$. For any $H \leq G, \underset{\sim}{\underset{\mathbf{X}}{\sim}}:=\operatorname{hocolim}_{\Lambda_{H}^{\text {op }}} \underset{\sim}{X}(K)$ is simply

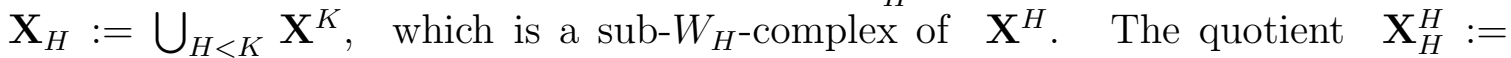
$\mathbf{X}^{H} / \mathbf{X}_{H}$ is the cofiber of the inclusion $j_{H}: \mathbf{X}_{H} \hookrightarrow \mathbf{X}^{H}$, which is a free pointed $W_{H}$-space (unless $\mathbf{X}_{H}=\emptyset$ ), with monoid action map $\zeta_{\mathbf{X}_{H}^{H}}^{*}: W_{H} \rightarrow \operatorname{haut}_{*}\left(\mathbf{X}_{H}^{H}\right)$ and $\Phi_{H}^{*}:=\mathbf{B} \zeta_{\mathbf{X}_{H}^{H}}^{*}: \mathbf{B} W_{H} \rightarrow \mathbf{B}$ haut $_{*}\left(\mathbf{X}_{H}^{H}\right)$. Evidently $\underline{\mathbf{X}}: \mathcal{F}_{\infty}=\mathcal{O}_{G}^{\text {op }} \rightarrow \mathcal{T}_{o p}$ realizes the Bredon homotopy action $\left\langle\left.\underline{\mathrm{X}}\right|_{\Lambda^{\mathrm{op}}},\left(\Phi_{H}^{*}\right)_{H \leq G}\right\rangle$ we have just defined (in all filtrations). In this case, we also say that the $G$-space $\mathbf{X}$ realizes $\left\langle\left.\underline{\mathrm{X}}\right|_{\Lambda^{\mathrm{op}}},\left(\Phi_{H}^{*}\right)_{H \leq G}\right\rangle$.

4.10. Definition. If $\underline{X}_{k}: \mathcal{F}_{k} \rightarrow \mathcal{T} o p$ realizes a Bredon homotopy action $\left.\underset{\sim}{X},\left(\Phi_{H}^{*}\right)_{H \leq G}\right\rangle$ in the $k$-th filtration, we may realize the pointed homotopy action $\Phi_{H}^{*}$ of $W_{H}$ on $\underset{\sim}{\mathbf{X}_{H}^{H}}$ by a topological pointed action of $W_{H}$ on a space $\underline{\mathbf{X}}_{H}^{H} \simeq \underset{\sim}{\mathbf{X}_{H}^{H}}$ (see Proposition 5.8 below). This fits into a homotopy cofibration sequence:

$$
\underline{\mathbf{X}}_{H} \stackrel{j_{H}}{\longrightarrow} \underset{\sim}{\sim}(H) \stackrel{q_{H}}{\longrightarrow} \underline{\mathbf{X}}_{H}^{H},
$$

where $\underline{\mathbf{X}}_{H} ;=\operatorname{hocolim}_{K>H} \underline{\mathrm{X}}_{k}(G / K) \quad$ (homotopic to (4.6) ). Note that the action of $N_{G} H$ on $\mathcal{F}_{k}$ by conjugation defines a $W_{H}$-action on $\underline{\mathbf{X}}_{H}$.

The $H$-lifting problem for $\underline{\mathrm{X}}_{k}$ is to find a map $\Psi_{H}$ making the following diagram commute up to homotopy: 


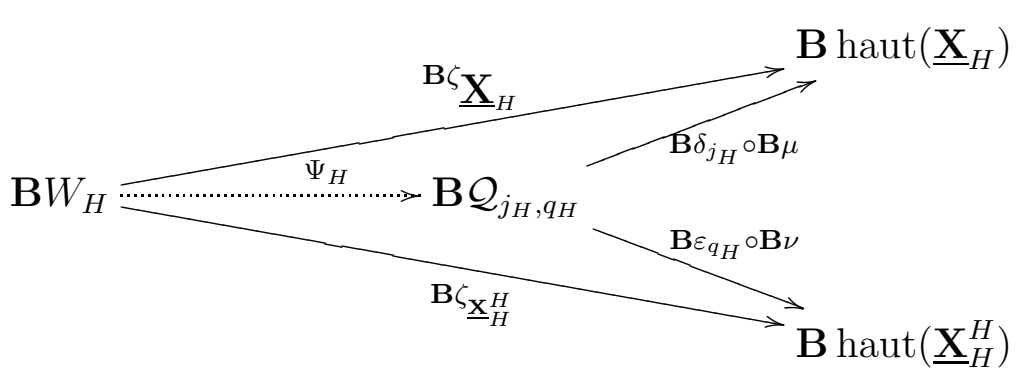

in the notation of (3.5).

We are now in a position to state our main result:

4.13. Theorem. A Bredon homotopy action $\mathcal{A}:=\left\langle\underset{\sim}{X},\left(\Phi_{H}^{*}\right)_{H \leq G}\right\rangle$ for a finite group $G$ can be realized by a $G$-space $\mathbf{X}$ if and only if one can inductively construct a coherent sequence of cofibrant diagrams $\left(\underline{X}_{k}: \mathcal{F}_{k} \rightarrow \mathcal{T} \text { op }\right)_{k=0}^{\infty} \quad$ realizing $\mathcal{A}$, where one can extend $\underline{X}_{k}$ to $\underline{X}_{k+1} \quad$ if and only if for each $\langle H\rangle \subseteq \widehat{\mathcal{F}}_{k+1} \backslash \widehat{\mathcal{F}}_{k}$, there is an $H \in\langle H\rangle$ for which the $H$-lifting problem (4.12) can be solved.

Proof. If $\mathcal{A}$ can be realized by a $G$-space $\mathbf{X}$, the corresponding diagrams $\underline{X}_{k} \quad$ were described in Example 4.9.

To see that solving the $H$-lifting problem suffices to extend an inductively-defined $\underline{\mathrm{X}}_{k}$ to $\underline{\mathrm{X}}_{k+1}$, we start with $\underline{\mathrm{X}}_{0}(G / G):=\underset{\sim}{X}(G) \quad$ (which we denote by $\mathbf{Y}$ ). To construct $\underline{\mathrm{X}}_{1}$, we must consider all maximal proper subgroups $M \in \widehat{\mathcal{F}}_{1}$, which are of two types:

(a) If $N_{G} M=M$, then $W_{M}=\{e\}$ and the correspondence $a M \mapsto M^{a}$ is a bijection between $G / M$ and $\langle M\rangle$. In this case we change $\mathbf{Y}=\underset{\sim}{X}(G / G) \rightarrow$ $\underset{\sim}{X}(G / M)$ into a cofibration $i: \mathbf{Y} \hookrightarrow \mathbf{Z}_{(M)} \quad$ (with no group action), and form a diagram consisting of a copy $i_{\left(M^{a}\right)}: \mathbf{Y} \hookrightarrow \mathbf{Z}_{\left(M^{a}\right)}$ of $i$ for each coset $M^{a} \in\langle M\rangle$, with $\underline{\mathrm{X}}_{1}\left(\left(\widetilde{\phi}_{a}^{M}\right)^{\mathrm{op}}\right)$ the homeomorphism identifying $\mathbf{Z}$ with $\mathbf{Z}_{\left(M^{a}\right)}$ (relative to the fixed subspace $\mathbf{Y}$ ).

(b) Otherwise $N_{G} M=G$, so $W_{M}=G / M$ and $\langle M\rangle$ is a singleton. We then apply Proposition and 3.6 to obtain a $W_{M}$-action on $\mathbf{Z}_{(M)} \simeq \underset{\sim}{X}(G / M)$, extending the trivial action on $\mathbf{Y}:=\underset{\sim}{X}(G / G)$. This is possible since we assume that the $M$-lifting problem can be solved. The action map $\zeta_{\mathbf{z}_{(M)}}$ : $G / M \rightarrow \operatorname{Aut}\left(\mathbf{Z}_{(M)}\right) \quad$ lifts to a $G$-action via the homomorphism $G \rightarrow G / M$.

Since all the conjugation $G$-actions we have described agree on $\mathbf{Y}$ (where they are trivial), we obtain a diagram $\underline{\mathrm{X}}_{1}: \mathcal{F}_{1} \rightarrow \mathcal{T}$ op, whose restriction to $\Lambda_{1}^{\mathrm{op}}$ consists of the inclusions $\mathbf{Y} \hookrightarrow \mathbf{Z}_{(M)}$ for all $M \in \widehat{\mathcal{F}}_{i} \backslash \widehat{\mathcal{F}}_{i-1}$.

At the $k$-th stage of the induction, we assume given a cofibrant diagram $\underline{X}_{k-1}$ : $\mathcal{F}_{k-1} \rightarrow \mathcal{T}$ op realizing $\underset{\sim}{X}$ up to filtration $k-1$. In particular, for each $H \in \widehat{\mathcal{F}}_{k} \backslash \widehat{\mathcal{F}}_{k-1}$ we have a space $\underline{X}_{H}:=\left(\underline{X}_{k-1}\right)_{H}$ as in $\underline{4.4}_{\text {, }}$, on which $N_{G} H$ acts (by conjugation), with $H \subseteq N_{G} H$ acting trivially. Thus $\underline{\mathrm{X}}_{H}$ has a $W_{H}$-action compatible with the structure maps of $\underline{X}_{k-1}$. 
For each conjugacy class $\langle H\rangle \subseteq \widehat{\mathcal{F}}_{k} \backslash \widehat{\mathcal{F}}_{k-1}$, we have a specified representative $H$. We use Proposition 5.8 to lift the given pointed homotopy action of $W_{H}$ on $\underset{\sim}{\mathbf{X}_{H}^{H}}$ to a (free) pointed action on $\mathbf{X}_{H}^{H} \simeq{\underset{\sim}{\sim}}_{H}^{H}$. Next, use Proposition 3.6 to produce a $W_{H^{-}}$ interpolation of the given $W_{H^{-}}$actions on $\underline{\mathrm{X}}_{H}$ and $\mathbf{X}_{H}^{H}$ for the homotopy cofibration sequence (4.6). Denote the new $W_{H}$-space we have produced by $\mathbf{Z}_{(H)} \simeq X(G / H)$. By Proposition [3.10, we may assume that the inclusion $i_{(H)}: \underline{X}_{H} \hookrightarrow \widetilde{\mathbf{Z}}_{(H)}$ is $W_{H}$-equivariant (with respect to the given conjugation action on $\underline{X}_{k-1}$ ).

For any conjugate $H^{a} \in\langle H\rangle$, choose a fixed element $a \in G$ representing the coset $a N_{G} H \in G / N_{G} H \cong\langle H\rangle$, and let $\underline{\mathrm{X}}_{k}\left(G / H^{a}\right):=\mathbf{Z}_{(H)}$. The $W_{H^{a} \text { action }}$ on $\underline{\mathrm{X}}_{k}\left(G / H^{a}\right)$ is the composite of the action map $W_{H} \rightarrow \operatorname{Aut}\left(\mathbf{Z}_{(H)}\right)$ with the isomorphism $\left(\rho_{a}^{H}\right)_{*}^{-1}: W_{H^{a}} \rightarrow W_{H} \quad$ induced by $\rho_{a}^{H}: N_{G} H \rightarrow N_{G} H^{a}$ (conjugation by $a$ ).

We define $i_{H^{a}}: \underline{\mathrm{X}}_{H^{a}} \hookrightarrow \mathbf{Z}_{(H)}$ to be the composite $i_{(H)} \circ\left(\widetilde{\phi}_{a}^{H}\right)^{\mathrm{op}}$. This is $W_{H^{a-}}$ equivariant because $\left(\widetilde{\phi}_{a}^{H}\right)^{\text {op }}$ is induced by $\rho_{a}^{H}$, so we have extended $\underline{X}_{k-1}$ to a diagram $\underline{\mathrm{X}}_{k}: \mathcal{F}_{k} \rightarrow \mathcal{T}$ op.

At the end of the process we have a full $\mathcal{O}_{G}^{\text {op }}$ diagram $\underline{\mathrm{X}}_{\infty}:=\operatorname{colim}_{k \rightarrow \infty} \underline{\mathrm{X}}_{k}$, and thus (by Theorem 1.6) a $G$-space $\mathbf{X}$ realizing the given Bredon homotopy action $\mathcal{A}$.

Note that homotopic maps $\Phi^{*} \sim\left(\Phi^{\prime}\right)^{*}: \mathbf{B} W_{H} \rightarrow \mathbf{B}$ haut ${ }_{*}\left(\underset{\mathbf{X}_{H}^{H}}{\sim_{H}}\right)$ induce pointed Borel $W_{H^{-}}$equivalences $\mathbf{X}_{H}^{H} \rightarrow\left(\mathbf{X}^{\prime}\right)_{H}^{H} \quad$ (assuming both are $W_{H}$-CW complexes), and homotopic lifts $\Psi \sim \Psi^{\prime}: \mathbf{B} W_{H} \rightarrow \mathbf{B} \mathcal{Q}_{j_{H}, q_{H}}$ in (4.12) yield Borel equivalent $W_{H}$-spaces $\mathbf{Z}_{H}$ and $\mathbf{Z}_{H}^{\prime}$, which implies that we have a weak equivalence of the resulting $\mathcal{F}_{k+1}$-diagrams $\underline{X}_{k+1}$ and $\underline{X}_{k+1}^{\prime}$, since all the structure maps which are not inclusions can be described in terms of the conjugation action of $G$.

4.14. Definition. If $\underline{\mathrm{X}}_{k}: \mathcal{F}_{k} \rightarrow \mathcal{T}$ op realizes a Bredon homotopy action $\mathcal{A}$ in the $k$-th filtration, for each conjugacy class $\langle H\rangle \subseteq \widehat{\mathcal{F}}_{k+1} \backslash \widehat{\mathcal{F}}_{k}$, choose any representative $H \in\langle H\rangle$. The $\langle H\rangle$-sequence of obstructions $\left(e_{n}\right)_{n=1}^{\infty}$ to extending $\underline{\mathrm{X}}_{k}$ to $\underline{\mathrm{X}}_{k+1}$ is defined by letting $e_{n} \in H^{n+2}\left(W_{H} ; \pi_{n+1} F\right)$ denote the $n$-th obstruction of Proposition 3.14 for the $H$-lifting problem (4.12).

The difference obstructions $f_{n} \in H^{n+1}\left(W_{H} ; \pi_{n+1} F\right)$ for distinguishing between different extensions of $\underline{\mathrm{X}}_{k}$ to $\underline{\mathrm{X}}_{k+1}$ are defined analogously.

4.15. Corollary. For any finite group $G$, a Bredon homotopy action $\mathcal{A}$ can be realized by a G-space $\mathbf{X}$ if and only if for each $k \geq 0$ and $\langle H\rangle \subseteq \widehat{\mathcal{F}}_{k+1} \backslash \widehat{\mathcal{F}}_{k}$, (some branch of) the inductively defined $\langle H\rangle$-sequence of obstructions $\left(e_{n}\right)_{n=1}^{\infty}$ vanishes. Moreover, two such realizations $\mathbf{X}$ and $\mathbf{X}^{\prime} \quad$ (by $G$-CW complexes) are $G$-homotopy equivalent if the corresponding sequence of difference obstructions vanish.

Remark 3.15 applies here too, of course.

4.16. Generalizations. The procedure described above extends to some infinite groups $G$, as long as we have a class function $\ell: \Lambda \rightarrow \kappa$ into some ordinal $\kappa$ with $\ell(K) \preceq \ell(H)$ for $H \leq K$. In this case we have a filtration corresponding to (4.3) of length $\kappa$, and thus a transfinite inductive procedure as in the proof of Theorem 4.13 . 
For example, if $G=\mathbb{Z}$ then $\ell: \Lambda \rightarrow \omega+1$ assigns to $n \mathbb{Z} \leq \mathbb{Z}$ the number of (not necessarily distinct) prime factors of $n$, with $\ell(\{0\})=\omega$. On the other hand, there is no such function $\ell$ for $G=\mathbb{Z}^{2}$ or $S^{1}$.

4.17. Some simple examples. The approach to realizing homotopy actions described here is quite complicated, in general, even for cyclic groups. Nevertheless, in certain cases the theory simplifies to some extent:

I. In a semi-free action all fixed points are global. In terms of a Bredon homotopy action this implies that the maps $j_{H}: \underset{\sim}{\mathbf{X}} \rightarrow \underset{\sim}{X}(H)$ are homotopy equivalences for $\{e\} \neq H$, and thus $\underset{\sim}{\mathbf{X}_{H}^{H}}$ is contractible - but $\underset{\sim}{X}(G)=: \mathbf{Y}$ need not be contractible. However, we do have a trivial $G$-action on $\mathbf{Y}$. Thus we are left with the obstructions of Proposition 3.14 for interpolating the given $G$-actions in the homotopy cofibration sequence $\mathbf{Y} \rightarrow \underset{\sim}{X}(\{e\}) \rightarrow \mathbf{Z}$. If these vanish, we obtain the required semi-free $G$-action an a space $\mathbf{X} \simeq \underset{\sim}{X}(\{e\})$.

Note that in this case $G$ need not be finite, so the examples mentioned in $\$ 2.19$ are relevant here.

II. A necessary condition in order for our obstruction theory to be effectively computable is that the (homotopy groups of) the spaces of self-equivalences of $\underset{\sim}{\mathbf{X}}$ and $\underset{\sim}{\mathbf{X}_{H}^{H}}$ in (4.6) are known for each $H \leq G$.

One simple case where this holds is when each of the above spaces is an EilenbergMac Lane space (cf. (2.20) $)$. If the groups $\pi_{*}$ haut $(\underset{\sim}{X}(H))$ are know - e.g., if $\underset{\sim}{X}(H)$ is also an Eilenberg-Mac Lane space - then the homotopy groups of

$$
\operatorname{Map}(\underset{\sim}{\mathbf{X}}, \underset{\sim}{X}(H)) \quad \text { and } \quad \operatorname{Map}\left(\underset{\sim}{X}(H), \underset{\sim}{\mathbf{X}_{H}^{H}}\right)
$$

are known (by [T]), so we may determine the homotopy groups of $\mathcal{P}_{j_{H}}$ and $\mathcal{P}_{q_{H}}$ up to an extension from the pullback diagram (2.6), (2.20), and (4.18), respectively, and these determine the homotopy groups of $\mathcal{Q}_{j_{H}, q_{H}}-$ and thus of the fibers $F-$ up to extensions from and (3.5) and (3.13).

III. The discussion above can also be extended to the case of two-stage Postnikov systems (see \$2.19 and Example 3.16 above).

\section{Appendix: Pointed homotopy aCtions}

For convenience, we collect here some basic facts about pointed homotopy actions. These are well-known, but we have not found a suitable reference in the literature.

5.1. Definition. A pointed homotopy action of a group $G$ on a pointed space $\mathbf{X}_{*}=$ $\left(\mathbf{X}, x_{0}\right)$ is (the homotopy class of) a map $\Phi^{*}: \mathbf{B} G \rightarrow$ haut $_{*}\left(\mathbf{X}_{*}\right)$. It is realized by a pointed $G$-action $\varphi_{\mathbf{Y}_{*}^{*}}^{*}: G \rightarrow \operatorname{Aut}_{*}\left(\mathbf{Y}_{*}\right)$ if there is a (pointed) homotopy equivalence $h: \mathbf{X}_{*} \rightarrow \mathbf{Y}_{*}$ such that 


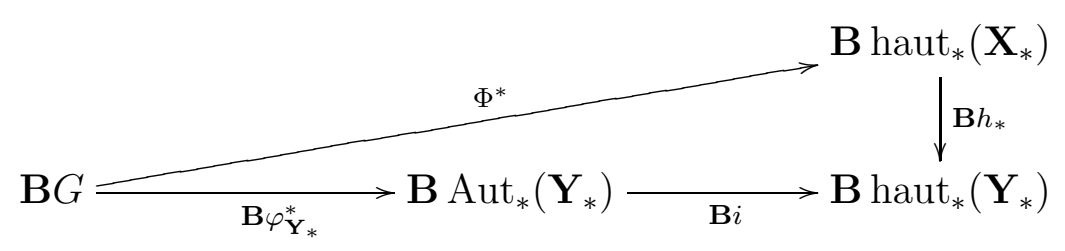

commutes up to homotopy.

5.3. Definition. A $G$-action $\varphi: G \rightarrow \operatorname{Aut}(\mathbf{X})$ on a space $\mathbf{X}$ lifts weakly to a pointed action $\varphi^{*}: G \rightarrow \operatorname{Aut}_{*}\left(\mathbf{Y}_{*}\right)$ if we have Borel $G$-equivalences $p: \mathbf{Z} \rightarrow \mathbf{X}$ and $p^{\prime}: \mathbf{Z} \rightarrow \mathbf{Y}$, with sections $i: \mathbf{X} \rightarrow \mathbf{Z}$ and $i^{\prime}: \mathbf{Y} \rightarrow \mathbf{Z}$ as in Lemma 1.9, such that the diagram of associative topological monoids (and multiplicative maps):

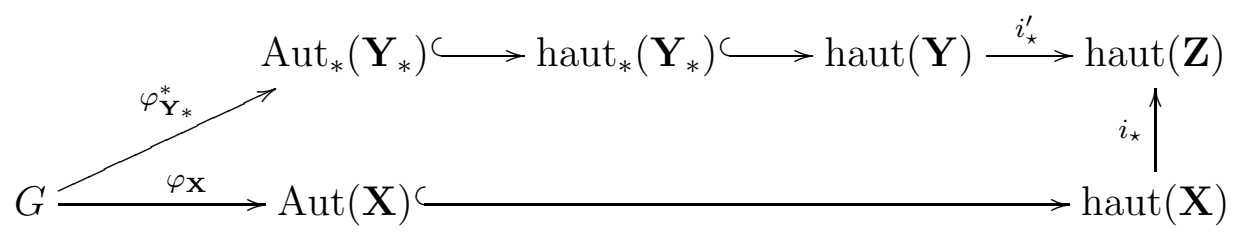

commutes up to homotopy after inverting the homotopy equivalence $i_{\star}$.

Since we assumed that $\mathbf{X}, \mathbf{Y}$, and $\mathbf{Z}$ are CW complexes, any homotopy equivalence between them can be made into a pointed homotopy equivalence by choosing appropriate (non-degenerate) base-points (cf. [Do, Theorem 3.6]). As a result, we may assume that $\mathbf{X}$ and $\mathbf{Y}$ in Definition 5.3 are pointed.

If $\mathbf{X}_{*}=\left(\mathbf{X}, x_{0}\right)$ is a $G$-space with chosen base point $x_{0}$, and the $G$-action is free on $\mathbf{X} \backslash\left\{x_{0}\right\}$, we call $\mathbf{X}_{*}$ a free pointed $G$-space. For any pointed $G$-space, the associated free pointed $G$-space is the quotient

$$
\mathbf{E} G \ltimes \mathbf{X}:=\mathbf{E} G \times \mathbf{X} / \mathbf{E} G \times\left\{x_{0}\right\},
$$

with $G$-action induced from the diagonal action on $\mathbf{E} G \times \mathbf{X}$. A homotopy fixed point for a $G$-space $\mathbf{X}$ is a $G$-map $f: \mathbf{E} G \rightarrow \mathbf{X}$. and we have:

5.5. Lemma. Any pointed $G$-space $\mathbf{X}_{*}$ has a $G$-map $\quad r: \mathbf{E} G \ltimes \mathbf{X} \rightarrow \mathbf{X}$ which is a pointed homotopy equivalence; if $\mathbf{X}_{*}$ is a free pointed $G$-space, the map $r$ is a G-homotopy equivalence.

Proof. We have a diagram of $G$-spaces:

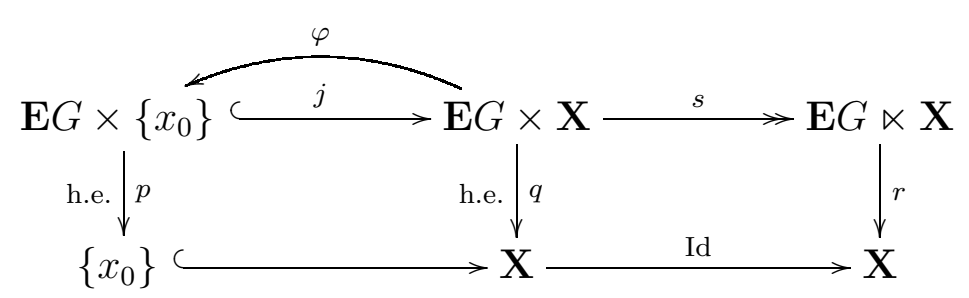

where the vertical maps are projections onto the second factor. Since each row is a cofibration sequence and $p$ and $q$ are Borel $G$-equivalences, so is $r$. If the pointed action on $\mathbf{X}_{*}$ is free, $r$ induces homotopy equivalences on all fixed point sets (which consist only of the basepoint for all $\{e\} \neq H \leq G$ ), so by [JS, Theorem (1.1)] $r$ is in fact a $G$-homotopy equivalence. 
5.7. Lemma. A G-space $\mathbf{X}$ with action $\varphi: G \rightarrow \operatorname{Aut}(\mathbf{X})$ has a homotopy fixed point corresponding to each weak lift of $\varphi$ to a pointed action $\varphi^{*}: G \rightarrow \operatorname{Aut}_{*}\left(\mathbf{Y}_{*}\right)$.

Proof. A weak lift of $\varphi$ to a pointed action $\varphi^{*}: G \rightarrow \operatorname{Aut}_{*}\left(\mathbf{Y}_{*}\right)$ yields a fixed point $y_{0} \in \mathbf{Y}$, and thus a homotopy fixed point for $\mathbf{Y}_{*}=\left(\mathbf{Y}, y_{0}\right)$ given by the constant map $c_{y_{0}}: \mathbf{E} G \rightarrow \mathbf{Y}$ (which is a $G$-map). This lifts to a homotopy fixed point $\hat{f}:\left(\mathrm{Id}, c_{y_{0}}\right): \mathbf{E} G \rightarrow \mathbf{E} G \times \mathbf{Y}$. Since $\mathrm{Id} \times h: \mathbf{E} G \times \mathbf{Z} \rightarrow \mathbf{E} G \times \mathbf{Y}$ is a $G$-map of free $G$-CW complexes (\$1.7) which is also a homotopy equivalence, it is actually a $G$-homotopy equivalence by [JS, Theorem (1.1)], with $G$-inverse $h^{-1}: \mathbf{E} G \times \mathbf{Y} \rightarrow$ $\mathbf{E} G \times \mathbf{Z}$. The $G$-map $k \circ h^{-1} \circ \hat{f}: \mathbf{E} G \rightarrow \mathbf{X}$ is the corresponding homotopy fixed point for $\mathbf{X}$.

Conversely, if $f: \mathbf{E} G \rightarrow \mathbf{X}$ is a $G$-map, we may factor $f$ in the model category $G$-Top (see \$1.5) as a $G$-cofibration followed by a $G$-fibration weak equivalence: $\mathbf{E} G \stackrel{\tilde{f}}{\rightarrow} \mathbf{Z} \stackrel{p}{\rightarrow} \mathbf{X}, . \quad$ If we let $\mathbf{Y}:=\mathbf{Z} / \mathbf{E} G$ denote the (homotopy) cofiber of $\tilde{f}$, with quotient $G$-map $p^{\prime}: \mathbf{Z} \rightarrow \mathbf{Y}$, then $\mathbf{Y}$ has a basepoint $y_{0}$ (corresponding to $\mathbf{E} G \subseteq \mathbf{Z}$ ), fixed under the $G$-action, and $p^{\prime}$ is a Borel $G$-equivalence since $\mathbf{E} G$ is contractible. Thus the $G$-action on $\mathbf{Y}_{*}=\left(\mathbf{Y}, y_{0}\right)$ yields the required pointed lift.

5.8. Proposition. Any pointed homotopy action $\Phi^{*}: \mathbf{B} G \rightarrow \mathbf{B}$ haut $_{*}\left(\mathbf{X}_{*}\right)$ can be realized by a (free) pointed G-action.

Proof. Pulling back the universal fibration $\mathbf{B} j$ of (1.2) along $\Phi:=i^{\prime} \circ \Phi^{*}$ yields the following (homotopy) pullback square:

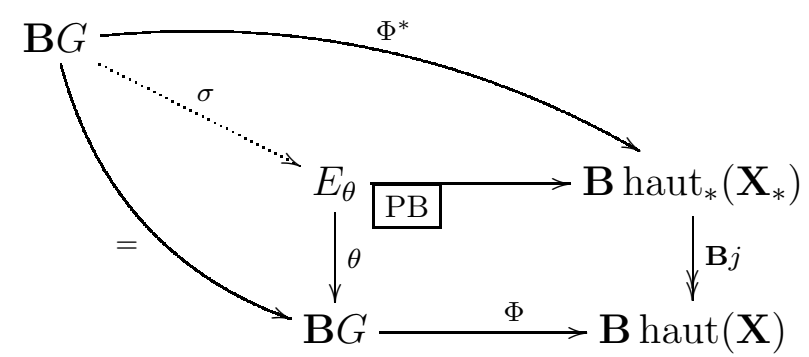

so we can use $\Phi^{*}$ to obtain a homotopy section $\sigma: \mathbf{B} G \rightarrow E_{\theta}$ as indicated.

We now use the lower left homotopy pullback square in (1.3) to obtain a homotopy fixed point $f: \mathbf{E} G \rightarrow \mathbf{X}_{1}$ :

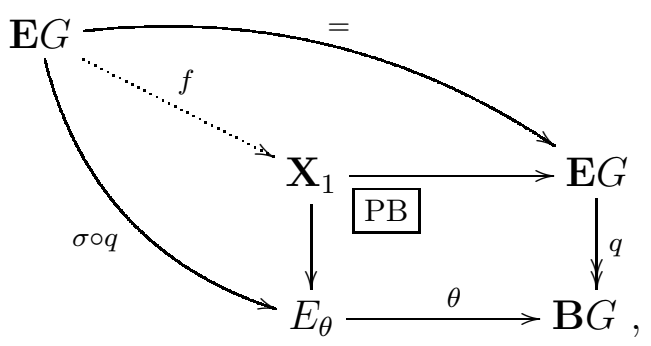

where $\mathbf{X}_{1} \simeq \mathbf{X}$. Hence by Lemma 5.7 we obtain a pointed $G$-action on a pointed space $\mathbf{Y}_{*}$ homotopy equivalent to $\mathbf{X}$. 


\section{REFERENCES}

[A] G. Allaud, "On the classification of fiber spaces", Math. Z. 92 (1966), pp. 110-125.

[BGM] M.G. Barratt, V.K.A.M. Gugenheim, \& J.C. Moore, "On semi-simplicial fibre bundles", Amer. J. Math. 81 (1959), pp. 639-657.

[BJT] D. Blanc, M.W. Johnson, \& J.M. Turner, "On realizing diagrams of П-algebras", Alg. E Geom. Top. 6 (2006), pp. 763-807.

[Bo] P.I. Booth, "Equivalent homotopy theories and groups of self-equivalences", in R.A. Piccinini, ed., Groups of Self-Equivalences and related topics (Montreal, PQ, 1988), Lec. Notes Math. 1425, Springer, Berlin-New York, 1990, pp. 1-16.

[Br] G.E. Bredon, Equivariant Cohomology Theories Springer-Verlag Lec. Notes Math. 34, Berlin-New York, 1967.

[C] G.E. Cooke, "Replacing Homotopy Actions by Topological Actions", Trans. AMS 237 (1978), pp. 391-406.

[Di] G. Didierjean, "Homotopie de l'espace des equivalences d'homotopie", Trans. AMS 330 (1992), pp. 153-163.

[tD] T. tom Dieck, Transformation Groups, De Greuter, Berlin-New York, 1987.

[Do] A. Dold, Halbexakte Homotopiefunktoren, Lec. Notes Math. 12, Springer, Berlin-New York, 1966.

[DL] A. Dold \& R.K. Lashof, "Principal quasifibrations and fibre homotopy equivalence of bundles", Ill. J. Math. 3 (1959), pp. 285-305.

[DDK] E. Dror-Farjoun, W.G. Dwyer \& D.M. Kan, "Equivariant maps which are self homotopy equivalences", Proc. AMS 80 (1980), pp. 670-672.

[DFZ] E. Dror-Farjoun \& A. Zabrodsky", "Unipotency and nilpotency in homotopy equivalences", Topology 18 (1979), pp. 187-197.

[DK1] W.G. Dwyer \& D.M. Kan, "Singular functors and realization functors", Proc. Kon. Ned. Akad. Wet. 2 (1984), pp. 147-153,

[DK2] W.G. Dwyer \& D.M. Kan, "Reducing Equivariant Homotopy Theory to the Theory of Fibrations", in R.A. Piccinini \& D. Sjerve, eds., Conference on Algebraic Topology in Honor of Peter Hilton (St. John's, Nfld., 1983), AMS, Providence, RI, 1985, pp. 35-49.

[DK3] W.G. Dwyer \& D.M. Kan, "Equivalences between homotopy theories of diagrams", in W. Browder, ed., Algebraic Topology and Algebraic K-Theory, Princeton U. Press, Princeton, NJ, 1987, pp. 180-205.

[DKS1] W.G. Dwyer, D.M. Kan, \& J.H. Smith, "An obstruction theory for simplicial categories", Proc. Kon. Ned. Akad. Wet. - Ind. Math. 89 (1986) No. 2, pp. 153-161.

[DKS2] W.G. Dwyer, D.M. Kan, \& J.H. Smith, "Homotopy commutative diagrams and their realizations", J. Pure \& Appl. Alg., 57 (1989), pp. 5-24.

[DM] W.G. Dwyer \& J.M. Møller, "Homotopy fixed points for cyclic $p$-group actions", Proc. AMS 125 (1997), pp. 3735-3739.

[DW] W.G. Dwyer \& C.W. Wilkerson, Homotopy fixed-point methods for Lie groups and finite loop spaces", Ann. Math. (2) 139 (1994), pp. 395-442.

[DZ] W.G. Dwyer \& A. Zabrodsky", "Maps between classifying spaces", in J. Aguadé \& R.M. Kane, eds., Algebraic Topology - Barcelona 1986, Lec. Notes Math. 1298, Springer, Berlin-New York, 1989, pp. 106-119.

[E] A.D. Elmendorf, "Systems of fixed point sets", Trans. AMS 277 (1983), pp. 275-284.

[F1] M. Fuchs, "Verallgemeinerte Homotopie-Homomorphismen und klassifizierende Raüme", Math. An. 161 (1965), 197-230.

[F2] M. Fuchs, "A modified Dold-Lashof construction that does classify $H$-principal fibrations", Math. An. 192 (1971), 328-340.

[GJ] P.G. Goerss \& J.F. Jardine, Simplicial Homotopy Theory, Progress in Mathematics 179, Birkhäuser, Basel-Boston, 1999.

[GS] J. Grodal \& J.H. Smith, "Classification of homotopy G-actions on spheres", in preparation.

[Ha1] V.L. Hansen, "The homotopy problem for the components in the space of maps on the n-sphere", Quart. J. Math., Ox. (2) 25 (1974), pp. 313-321. 
[Ha2] V.L. Hansen, "The homotopy groups of a space of maps between oriented closed surfaces", Bull. LMS 15 (1983), pp. 360-364.

[Hi] P.S. Hirschhorn, Model Categories and their Localizations, Math. Surveys \& Monographs 99, AMS, Providence, RI, 2002.

[I] S. Illman, Equivariant singular homology and cohomology, I, Memoirs AMS 1 (No. 156), AMS, Providence, RI, 1975.

[JMO] S. Jackowski, J.E. McClure, \& R. Oliver, "Homotopy classification of self-maps of $B G$ via G-actions, Parts I, II", Ann. Math. (2) 135 (1992), pp. 183-226, 227-270.

[JS] I.M. James \& G.B. Segal, "On equivariant homotopy type", Topology 17 (1978), pp. 267272 .

[La] J. Lannes, "Sur les espaces fonctionnels dont la source est le classifiant d'une $p$-groupe abélien élémentaire", Pub. Math. IHES 75 (1992), pp. 135-244.

[Ma1] J.P. May, Simplicial Objects in Algebraic Topology, U. Chicago, Chicago-London, 1967.

[Ma2] J.P. May, Classifying spaces and fibrations, Mem. AMS 155, AMS, Providence, RI, 1975.

[Mo] J.M. Møller. "Self-homotopy equivalences of group cohomology spaces", J. Pure E Appl. Alg., 73 (1991), pp. 23-37.

[O] J. Oprea, "Lifting homotopy actions in rational homotopy theory", J. Pure Appl. Alg. 32 (1984), pp. 177-190.

[Pi] R.J. Piacenza, "The homotopy theory of diagrams", in J.P. May, ed., Equivariant homotopy and cohomology theory, Reg. Conf. Ser. Math. 91, AMS, Providence, RI, 1996, pp. 47-56.

[Pr] M. Prezma, "Homotopy Normal Maps", Alg. Geom. Top. 12 (2012), pp. 1211-1238.

[R] C.A. Robinson, "Moore-Postnikov systems for non-simple fibrations", Ill. J. Math. 16 (1972), pp. 234-242.

[SV1] R. Schwänzl \& R.M. Vogt, "Coherence in homotopy group actions", in S. Jackowski \& K. Pawałowski, eds., Transformation Groups -Poznán 1985, Springer-Verlag Lec. Notes Math. 1217, Berlin-New York, 1986, pp. 364-390.

[SV2] R. Schwänzl \& R.M. Vogt, "The categories of $A_{\infty^{-}}$and $E_{\infty^{-}}$-monoids and ring spaces as closed simplicial and topological model categories", Arch. Math. 56 (1991), pp. 405-411.

[PSm] P.A. Smith, "Fixed-point theorems for periodic transformations", Amer. J. Math. 63 (1941), pp. 1-8.

[LSm] L.E. Smith, "Replacing homotopy actions by topological actions, II", Trans. AMS 317 (1990), pp. 1-20.

[St] J. D. Stasheff, "A classification theorem for fibre spaces", Prop. AMS 7 (1963), pp. 239-246

[Su] D.P. Sullivan, "Geometric Topology, Part I. Localization, periodicity, and Galois symmetry", Springer-Verlag, Berlin-New York, 2005 [reprint of the 1970 MIT notes].

[T] R. Thom, 'L'homologie de espaces fonctionnels', in Colloque de Topologie Algébrique (Louvain 1956), G. Thone, Liège Masson, Paris, 1957, pp. 29-39.

[Z] A. Zabrodsky, 'On George Cooke's theory of homotopy and topological actions, in R.M. Kane, S.O. Kochman, P.S. Selick, \& V.P. Snaith, eds., Current Trends in Algebraic Topology, Part 2 (London, Ont., 1981), CMS Conf. Proc. 2, AMS, Providence, RI, 1982, pp. 313-317.

Department of Mathematics, University of Haifa, 31905 Haifa, IsRAel

E-mail address: blanc@math.haifa.ac.il, sen_deba@math.haifa.ac.il 